\title{
The representation category of any compact group is the bimodule category of a $\mathrm{II}_{1}$ factor
}

\author{
Journal für die reine und angewandte Mathematik (Crelle's Journal) 643 (2010), 171-199.
}

By SÉbastien FAlguières ${ }^{(1,2)}$ And StefaAn VAeS ${ }^{(1,2)}$

\begin{abstract}
We prove that given any compact group $G$, there exists a minimal action of $G$ on a $\mathrm{II}_{1}$ factor $M$ such that the bimodule category of the fixed-point $\mathrm{II}_{1}$ factor $M^{G}$ is naturally equivalent with the representation category of $G$. In particular, all subfactors of $M^{G}$ with finite Jones index can be described explicitly.
\end{abstract}

\section{Introduction and statements of main results}

One of the richest invariants of a $\mathrm{II}_{1}$ factor $P$ is the bimodule category $\operatorname{Bimod}(P)$ consisting of all $P$-P-bimodules ${ }_{P} \mathcal{H}_{P}$ (see $\left.[3,15]\right)$ of finite Jones index: $\operatorname{dim}\left({ }_{P} \mathcal{H}\right)<\infty$ and $\operatorname{dim}\left(\mathcal{H}_{P}\right)<\infty$. Equipped with the Connes tensor product, $\operatorname{Bimod}(P)$ is a $\mathrm{C}^{*}$-tensor category. Note that $\operatorname{Bimod}(P)$ contains both the fundamental group $\mathcal{F}(P)$ and the outer automorphism group $\operatorname{Out}(P)$, because every $*$-isomorphism $\pi: P \rightarrow p P p$ yields the bimodule ${ }_{P} \mathrm{~L}^{2}(P) p_{\pi(P)}$. More precisely, the group-like elements in $\operatorname{Bimod}(P)$ form an extension of $\mathcal{F}(P)$ by $\operatorname{Out}(P)$. Moreover, $\operatorname{Bimod}(P)$ encodes, in a certain sense, all subfactors $P_{0} \subset P$ of finite Jones index [8]: performing Jones' basic construction, we get $P_{0} \subset P \subset P_{1}$ and obtain the $P$-P-bimodule ${ }_{P} \mathrm{~L}^{2}\left(P_{1}\right)_{P}$. As a result, it seemed until recently quite hopeless to explicitly compute $\operatorname{Bimod}(P)$ for any $\mathrm{II}_{1}$ factor $P$.

But, in $[10,11,12]$, Sorin Popa obtained several breakthrough rigidity results for $\mathrm{II}_{1}$ factors, which allowed in particular to compute invariants like $\mathcal{F}(P)$ and $\operatorname{Out}(P)$ for concrete $\mathrm{II}_{1}$ factors $P$. Without being exhaustive, we mention the following results: in [12], Popa obtained the first $\mathrm{II}_{1}$ factors having trivial fundamental group, while in [10], he constructed examples with prescribed countable fundamental group. Very recently, Popa and the second author $[17,16]$ proved that the invariant $\mathcal{F}(P)$ actually ranges over a large family of uncountable subgroups of $\mathbb{R}_{+}$. In [7], Ioana, Peterson and Popa proved the existence of $\mathrm{II}_{1}$ factors $P$ such that $\operatorname{Out}(P)$ is any prescribed second countable compact abelian group. In particular, this settled the long standing open problem of the possible existence of $\mathrm{II}_{1}$ factors only having inner automorphisms. The first concrete computations of $\operatorname{Out}(P)$ were given by Popa and the second author in [18] and later refined in [20]. On the other hand, we proved in [5] that also all non-abelian compact groups arise as $\operatorname{Out}(P)$.

The $\mathrm{II}_{1}$ factors studied in [7] are amalgamated free products $M=M_{0} *_{N} M_{1}$ (see Section 2.6 for definitions). The main result of [7] says that a von Neumann subalgebra $Q$ of $M$ having the property (T) of Connes and Jones (see [4] and Section 2.10 below), or just having the relative property (T) in the sense of Popa [12], must essentially be contained in either $M_{0}$ or $M_{1}$. In particular, if $M_{0}$ has itself property $(\mathrm{T})$ and $M_{1}$ has, say, the Haagerup property, every automorphism of $M$ must preserve $M_{0}$ globally. This is the starting point to compute Out $(M)$ in certain particular cases, leading to the above mentioned results of $[5,7]$.

\footnotetext{
${ }^{1}$ Partially supported by ERC Starting Grant VNALG-200749 and Research Programme G.0231.07 of the Research Foundation - Flanders (FWO)

${ }^{2}$ Department of Mathematics; K.U.Leuven; Celestijnenlaan 200B; B-3001 Leuven (Belgium). E-mail: sebastien.falguieres@wis.kuleuven.be, stefaan.vaes@wis.kuleuven.be
} 
In [21], the scope of the methods of [7] was enlarged so that in certain cases not only Out $(M)$ but also $\operatorname{Bimod}(M)$ could actually be computed. The main result of [21] proves the existence of $\mathrm{II}_{1}$ factors $M$ having trivial bimodule category and hence also trivial subfactor structure, trivial fundamental group and trivial outer automorphism group. Note however that the results in $[5,7,21]$ are existence theorems. The first concrete $\mathrm{II}_{1}$ factors with trivial bimodule category were given in [20], which included as well concrete examples of $\mathrm{II}_{1}$ factors where $\operatorname{Bimod}(P)$ is a Hecke-like category.

We prove in this paper that the representation category of an arbitrary compact group $G$ can be realized as the bimodule category of a $\mathrm{II}_{1}$ factor. More precisely, we prove the existence of a minimal action $G \curvearrowright M$ of $G$ on a $\mathrm{II}_{1}$ factor $M$, such that the bimodule category $\operatorname{Bimod}\left(M^{G}\right)$ of the fixed point algebra $M^{G}$ can be identified with the representation category $\operatorname{Rep}(G)$. Note that $G \curvearrowright M$ is called minimal if $G \rightarrow \operatorname{Aut}(M)$ is injective and the fixed point algebra $M^{G}$ has trivial relative commutant in $M$, i.e. $M \cap\left(M^{G}\right)^{\prime}=\mathbb{C} 1$. Whenever $G \curvearrowright M$ is a minimal action, there is a natural embedding of $\operatorname{Rep}(G) \operatorname{into} \operatorname{Bimod}\left(M^{G}\right)$ (see [19] and Section 2.7). The striking point is that there exist minimal actions such that this embedding is surjective (up to unitary equivalence). As in $[5,7,21]$, our result is an existence theorem, involving a Baire category argument (Theorem 2.8).

As in $[5,7]$, the $\mathrm{II}_{1}$ factor $M$ in the previous paragraph is of the form $M=M_{0} *_{N} M_{1}$ and the action $G \curvearrowright M$ is such that $G$ acts trivially on $M_{0}$, leaves $M_{1}$ globally invariant and satisfies $M_{1}^{G}=N$. Our main theorem is the following.

Theorem 1.1. Let $G$ be a second countable compact group. There exists a $I I_{1}$ factor $M$ and a minimal action $G \curvearrowright M$ such that, writing $P:=M^{G}$, every finite index $P$-P-bimodule is isomorphic with ${ }_{P} \operatorname{Mor}\left(H_{\pi}, \mathrm{L}^{2}(M)\right)_{P}$ for a uniquely determined finite dimensional unitary representation $\pi$ : $G \rightarrow \mathcal{U}\left(H_{\pi}\right)$.

More precisely, $\operatorname{Rep}(G) \rightarrow \operatorname{Bimod}(P): \pi \mapsto{ }_{P} \operatorname{Mor}\left(H_{\pi}, \mathrm{L}^{2}(M)\right)_{P}$ defines an equivalence of $C^{*}$-tensor categories.

Theorem 1.1 provides examples of $\mathrm{II}_{1}$ factors for which all finite index bimodules over $P=M^{G}$ can be listed explicitly, labeled by the finite dimensional unitary representations of $G$. Since the category of finite index bimodules over $P$ encodes in a certain way all finite index subfactors of $P$, these can be explicitly listed as well. In particular, Jones' invariant [8]

$$
\mathcal{C}(P):=\left\{\left[P: P_{0}\right] \mid P_{0} \subset P \text { irreducible, finite index subfactor }\right\}
$$

can be explicitly computed for the $\mathrm{II}_{1}$ factors $P=M^{G}$ given by Theorem 1.1. The precise result goes as follows. We make use of Jones' tunnel construction [8, Corollary 3.1.9] saying that for every finite index inclusion of $\mathrm{II}_{1}$ factors $P \subset N$, there exists a finite index subfactor $P_{0} \subset P$ such that $P_{0} \subset P \subset N$ is the basic construction. Moreover, $P_{0}$ is uniquely determined up to unitary conjugacy in $P$.

Theorem 1.2. Let $G \stackrel{\sigma}{\curvearrowright} M$ be a minimal action of the second countable compact group $G$ on the $I I_{1}$ factor $M$ and write $P=M^{G}$. Assume that $\sigma$ satisfies the conclusion Theorem 1.1, meaning that every finite index $P$-P-bimodule is of the form ${ }_{P} \operatorname{Mor}\left(H_{\pi}, \mathrm{L}^{2}(M)\right)_{P}$ for some finite dimensional unitary representation $\pi$ of $G$.

Whenever $G \stackrel{\alpha}{\curvearrowright} A$ is an action on the finite dimensional von Neumann algebra $A$ with $\mathcal{Z}(A)^{G}=\mathbb{C} 1$, define the finite index subfactor $P(\alpha) \subset P$ such that $1 \otimes P(\alpha) \subset 1 \otimes P \subset(A \otimes M)^{\alpha \otimes \sigma}$ is the basic construction. Here $(\alpha \otimes \sigma)_{g}:=\alpha_{g} \otimes \sigma_{g}$ and we note that $P(\alpha)$ is uniquely defined up to unitary conjugacy in $P$. 
- Every finite index subfactor of $P$ is unitarily conjugate to one of the $P(\alpha)$.

- $[P: P(\alpha)]=\operatorname{dim} A$ and $P(\alpha) \subset P$ is irreducible iff $A^{G}=\mathbb{C} 1$.

- If $G \stackrel{\alpha}{\curvearrowright} A$ and $G \stackrel{\beta}{\curvearrowright} B$ satisfy $\mathcal{Z}(A)^{G}=\mathbb{C} 1$ and $\mathcal{Z}(B)^{G}=1$, then the subfactors $P(\alpha)$ and $P(\beta)$ of $P$ are unitarily conjugate in $P$ iff there exists a $*$-isomorphism $\pi: A \rightarrow B$ satisfying $\beta_{g} \circ \pi=\pi \circ \alpha_{g}$ for all $g \in G$.

In particular, the set of index values of irreducible finite index subfactors of $P$ is given by

$$
\mathcal{C}(P)=\left\{\operatorname{dim}(A) \mid A \text { finite dimensional von Neumann algebra, } G \curvearrowright A, A^{G}=\mathbb{C} 1\right\} .
$$

\section{Acknowledgment}

The authors would like to thank the referee for carefully reading the paper and making several suggestions that have lead to a considerable improvement of the exposition.

\section{Preliminaries}

\subsection{The *-algebra of operators affiliated with a $\mathrm{II}_{1}$ factor}

Let $M \subset \mathrm{B}(H)$ be a $\mathrm{II}_{1}$ factor with normal tracial state $\tau$. Denote by $\mathcal{M}$ the closed densely defined operators affiliated with $M$. By [9, Theorem XV, page 229], we know that $\mathcal{M}$ is a $*$-algebra, where sum and product are defined as the closure of sum and product on the natural domains and where the adjoint is the usual adjoint of operators. Denote by $\mathcal{M}^{+}$the positive self-adjoint operators affiliated with $M$. Then, $\tau$ has a natural extension to a positive-linear map $\mathcal{M}^{+} \rightarrow[0,+\infty]$. Define, for $x \in \mathcal{M},|x|:=\left(x^{*} x\right)^{1 / 2},\|x\|_{2}:=\tau\left(x^{*} x\right)^{1 / 2}$ and $\|x\|_{1}:=\tau(|x|)$. Put

$$
\mathrm{L}^{2}(M):=\left\{x \in \mathcal{M} \mid\|x\|_{2}<\infty\right\} \quad \text { and } \quad \mathrm{L}^{1}(M):=\left\{x \in \mathcal{M} \mid\|x\|_{1}<\infty\right\} .
$$

Actually, $\mathrm{L}^{1}(M)$ is the linear span of $\left\{x \in \mathcal{M}^{+} \mid \tau(x)<\infty\right\}$ and $\tau$ extends to a linear map $\mathrm{L}^{1}(M) \rightarrow \mathbb{C}$. Both $\mathrm{L}^{2}(M)$ and $\mathrm{L}^{1}(M)$ are stable under the adjoint and are $M$ - $M$-bimodules. Finally, the product of two elements in $\mathrm{L}^{2}(M)$ belongs to $\mathrm{L}^{1}(M)$, the Cauchy-Schwartz inequality holds and the scalar product $\langle x, y\rangle:=\tau\left(x^{*} y\right)$ turns $\mathrm{L}^{2}(M)$ into a Hilbert space.

Every $x \in \mathcal{M}$ has a unique polar decomposition, $x=u|x|$, where $u$ is a partial isometry in $M$ with $u^{*} u$ equal to the support projection of $|x|$. If $N \subset M$ is an irreducible subfactor, meaning that $N^{\prime} \cap M=\mathbb{C} 1$, every element $x \in \mathcal{M}$ satisfying $a x=x a$ for all $a \in N$, belongs to $\mathbb{C} 1$ as well. Indeed, if $x=u|x|$ is the polar decomposition of $x$, the uniqueness of the polar decomposition implies that $u$ and $|x|$ commute with all unitaries in $N$. Hence, $u$ is scalar. By the uniqueness of the spectral decomposition of $|x|$, all spectral projections of $|x|$ are scalar and hence, also $|x|$ follows scalar.

\subsection{Some notational conventions}

For any von Neumann algebra $M$ we denote $M^{n}:=\mathrm{M}_{n}(\mathbb{C}) \otimes M$. We write $\mathbb{C}^{n}\left(\mathbb{C}^{m}\right)^{*}$ instead of $\mathrm{B}\left(\mathbb{C}^{m}, \mathbb{C}^{n}\right)$. We implicitly consider $\mathbb{C}^{n}\left(\mathbb{C}^{m}\right)^{*}$ as a Hilbert space with scalar product $\langle\xi, \eta\rangle=\operatorname{Tr}\left(\xi^{*} \eta\right)$. Obviously, $\mathbb{C}^{n}\left(\mathbb{C}^{m}\right)^{*}$ is an $\mathrm{M}_{n}(\mathbb{C})-\mathrm{M}_{m}(\mathbb{C})$-bimodule. We denote by $e_{i} \in \mathbb{C}^{n}$ the natural vectors. All *-homomorphisms between von Neumann algebras are implicitly assumed to be normal. 


\subsection{The bimodule category and fusion algebra of a $\mathrm{II}_{1}$ factor}

Let $(M, \tau)$ be a von Neumann algebra with faithful normal tracial state and $\mathcal{H}_{M}$ a right Hilbert $M$-module. There exists a projection $p \in \mathrm{B}\left(\ell^{2}(\mathbb{N})\right) \bar{\otimes} M$ such that $\mathcal{H}_{M} \cong p\left(\ell^{2}(\mathbb{N}) \bar{\otimes} \mathrm{L}^{2}(M)\right)_{M}$ and this projection $p$ is uniquely defined up to equivalence of projections in $\mathrm{B}\left(\ell^{2}(\mathbb{N})\right) \bar{\otimes} M$. We denote $\operatorname{dim}\left(\mathcal{H}_{M}\right):=(\operatorname{Tr} \otimes \tau)(p)$. Observe that the number $\operatorname{dim}\left(\mathcal{H}_{M}\right)$ depends on the choice of tracial state $\tau$ in the non-factorial case. An $N$-M-bimodule ${ }_{N} \mathcal{H}_{M}$ is said to be of finite Jones index if $\operatorname{dim}\left({ }_{N} \mathcal{H}\right)<\infty$ and $\operatorname{dim}\left(\mathcal{H}_{M}\right)<\infty$. In particular, the Jones index of a subfactor $N \subset M$ is defined as $[M: N]:=\operatorname{dim}\left(\mathrm{L}^{2}(M)_{N}\right)$, see $[8]$.

We constantly use the following well known principle: if $N \subset M$ is a finite index subfactor and $Q \subset N$ a von Neumann subalgebra such that $Q^{\prime} \cap N$ is finite dimensional, then also $Q^{\prime} \cap M$ is finite dimensional (see e.g. [20, Lemma A.3]).

Let $M, N, P$ be von Neumann algebras with faithful normal tracial states and fix bimodules ${ }_{M} \mathcal{H}_{N}$ and ${ }_{N} \mathcal{K}_{P}$. We briefly recall the construction of the Connes tensor product ${ }_{M}\left(\mathcal{H} \otimes_{N} \mathcal{K}\right)_{P}$ and refer to $\left[3\right.$, V.Appendix B] for details. Denote by $\mathcal{H}^{0}$ the set of vectors $\xi \in \mathcal{H}$ such that the linear map $N \rightarrow \mathcal{H}: a \mapsto \xi a$ extends to a bounded operator $L_{\xi}: \mathrm{L}^{2}(N) \rightarrow \mathcal{H}$. Then, $\mathcal{H}^{0}$ is a dense subspace of $\mathcal{H}$. One defines an $N$-valued scalar product on $\mathcal{H}^{0}$ by setting $\langle\xi, \eta\rangle_{N}:=L_{\xi}^{*} L_{\eta}$. The Connes tensor product $\mathcal{H} \otimes_{N} \mathcal{K}$ is defined as the separation and completion of the algebraic tensor product $\mathcal{H}^{0} \otimes_{\text {alg }} \mathcal{K}$ for the scalar product

$$
\langle a \otimes \xi, b \otimes \eta\rangle:=\left\langle\xi,\langle a, b\rangle_{N} \eta\right\rangle .
$$

The Hilbert space $\mathcal{H} \otimes_{N} \mathcal{K}$ is turned into an $M$-P-bimodule in the following way:

$$
a \cdot(b \otimes \xi)=a b \otimes \xi \quad \text { and } \quad(b \otimes \xi) \cdot a=b \otimes(\xi a) .
$$

Whenever $p \in \mathrm{B}\left(\ell^{2}(\mathbb{N})\right) \bar{\otimes} N$ is a projection and $\psi: P \rightarrow p\left(\mathrm{~B}\left(\ell^{2}(\mathbb{N})\right) \bar{\otimes} N\right) p$ is a $*$-homomorphism, define the $N$-P-bimodule $H(\psi)$ on the Hilbert space $\left(\ell^{2}(\mathbb{N})^{*} \otimes \mathrm{L}^{2}(N)\right) p$ with left and right module actions given by

$$
a \cdot \xi:=a \xi \quad \text { and } \quad \xi \cdot b=\xi \psi(b) .
$$

Every $N$-P-bimodule is isomorphic with an $N$-P-bimodule of the form $H(\psi)$. Furthermore, if $\psi: P \rightarrow p\left(\mathrm{~B}\left(\ell^{2}(\mathbb{N})\right) \bar{\otimes} N\right) p$ and $\eta: P \rightarrow q\left(\mathrm{~B}\left(\ell^{2}(\mathbb{N})\right) \bar{\otimes} N\right) q$, then ${ }_{N} H(\psi)_{P} \cong{ }_{N} H(\eta)_{P}$ if and only if there exists $u \in \mathrm{B}\left(\ell^{2}(\mathbb{N})\right) \bar{\otimes} N$ satisfying $u u^{*}=p, u^{*} u=q$ and $\psi(a)=u \eta(a) u^{*}$ for all $a \in P$.

Whenever ${ }_{M} \mathcal{H}_{N}$ is an $M$-N-bimodule, the Connes tensor product ${ }_{M}\left(\mathcal{H} \otimes_{N} H(\psi)\right)_{P}$ is isomorphic with the $M$-P-bimodule defined on the Hilbert space $\left(\ell^{2}(\mathbb{N})^{*} \otimes \mathcal{H}\right) p$ with left and right module actions given by $a \cdot \xi=a \xi$ and $\xi \cdot b=\xi \psi(b)$. In particular, $H(\rho) \otimes_{N} H(\psi) \cong H((\operatorname{id} \otimes \rho) \psi)$.

In the previous two paragraphs, one can analogously describe bimodules by homomorphisms on the left: ${ }_{M} \mathcal{H}_{N} \cong{ }_{\varphi(M)} p\left(\ell^{2}(\mathbb{N}) \otimes \mathrm{L}^{2}(N)\right)_{N}$ for a $*$-homomorphism $\varphi: M \rightarrow p\left(\mathrm{~B}\left(\ell^{2}(\mathbb{N}) \bar{\otimes} N\right)\right) p$.

The contragredient of an $M$-N-bimodule ${ }_{M} \mathcal{H}_{N}$ is defined on the conjugate Hilbert space $\mathcal{H}^{*}$ with bimodule actions given by $a \cdot \xi^{*}:=\left(\xi a^{*}\right)^{*}$ and $\xi^{*} \cdot b:=\left(b^{*} \xi\right)^{*}$.

From now on, fix a $\mathrm{II}_{1}$ factor $M$. The category $\operatorname{Bimod}(M)$ consists of all finite index $M-M$ bimodules, with morphisms given by the $M$ - $M$-bimodular maps. We refer to [1] for background material and results on bimodules and fusion algebras, in particular in relation with subfactors. Every finite index $M$ - $M$-bimodule is isomorphic with an $H(\psi)$ for some finite index inclusion $\psi: M \rightarrow p M^{n} p$. 
One also defines the fusion algebra FAlg $(M)$ of $M$ as the set of finite index $M$ - $M$-bimodules modulo unitary equivalence. We recall that an abstract fusion algebra $\mathcal{A}$ is a free $\mathbb{N}$-module $\mathbb{N}[\mathcal{G}]$ equipped with the following additional structure:

- an associative and distributive product operation, and a multiplicative unit element $e \in \mathcal{G}$,

- an additive, anti-multiplicative, involutive map $x \mapsto \bar{x}$, called conjugation,

satisfying Frobenius reciprocity: defining the numbers $m(x, y ; z) \in \mathbb{N}$ for $x, y, z \in \mathcal{G}$ through the formula

$$
x y=\sum_{z} m(x, y ; z) z,
$$

one has $m(x, y ; z)=m(\bar{x}, z ; y)=m(z, \bar{y} ; x)$ for all $x, y, z \in \mathcal{G}$.

The base $\mathcal{G}$ of the fusion algebra $\mathcal{A}$ is canonically determined: these are exactly the non-zero elements of $\mathcal{A}$ that cannot be expressed as the sum of two non-zero elements. The elements of $\mathcal{G}$ are called the irreducible elements of the fusion algebra $\mathcal{A}$.

Two examples of fusion algebras arise as follows.

- Let $\Gamma$ be a group and define $\mathcal{A}=\mathbb{N}[\Gamma]$.

- Let $G$ be a compact group and define the fusion algebra $\operatorname{Rep}(G)$ as the set of equivalence classes of finite dimensional unitary representations of $G$. The operations on $\operatorname{Rep}(G)$ are given by direct sum and tensor product of representations.

We end with the following probably well known lemma. For convenience, we give a proof.

Lemma 2.1. Let $N \subset M$ be an irreducible inclusion of $I I_{1}$ factors and ${ }_{M} \mathcal{K}_{M}$ a finite index $M$ $M$-bimodule. Whenever ${ }_{N} \mathcal{H}_{N}$ is a finite index $N$-N-bimodule, the vector space of bounded $N-N$ bimodular operators from $\mathcal{H}$ to $\mathcal{K}$, is finite dimensional.

Proof. Write ${ }_{M} \mathcal{K}_{M} \cong{ }_{\psi(M)} p\left(\mathbb{C}^{n} \otimes \mathrm{L}^{2}(M)\right)_{M}$ and ${ }_{N} \mathcal{H}_{N} \cong{ }_{\gamma(N)} q\left(\mathbb{C}^{m} \otimes \mathrm{L}^{2}(N)\right)_{N}$ for some finite index inclusions $\psi: M \rightarrow p M^{n} p$ and $\gamma: N \rightarrow q N^{m} q$. Define

$$
\mathcal{L}=\left\{T \in p\left(\mathbb{C}^{n}\left(\mathbb{C}^{m}\right)^{*} \otimes M\right) q \mid \psi(a) T=T \gamma(a) \text { for all } a \in N\right\}
$$

For every $T \in \mathcal{L}$, left multiplication by $T$ defines an $N$-N-bimodular map $q\left(\mathbb{C}^{m} \otimes \mathrm{L}^{2}(N)\right) \rightarrow$ $p\left(\mathbb{C}^{n} \otimes \mathrm{L}^{2}(M)\right)$. Conversely, let $\theta: q\left(\mathbb{C}^{m} \otimes \mathrm{L}^{2}(N)\right) \rightarrow p\left(\mathbb{C}^{n} \otimes \mathrm{L}^{2}(M)\right)$ be a bounded $N$ - $N$-bimodular operator. Define $T \in p\left(\mathbb{C}^{n}\left(\mathbb{C}^{m}\right)^{*} \otimes \mathrm{L}^{2}(M)\right) q$ by the formula

$$
T=\sum_{i=1}^{m} \theta\left(q\left(e_{i} \otimes 1\right)\right)\left(e_{i}^{*} \otimes 1\right)
$$

It follows that $\psi(a) T=T \gamma(a)$ for all $a \in N$. Define $A:=p M^{n} p \cap \psi(N)^{\prime}$. Since $N \subset M$ is irreducible and $\psi(M) \subset p M^{n} p$ has finite index, $A$ is finite dimensional. The operator $T T^{*}$ belongs to $p\left(\mathrm{M}_{n}(\mathbb{C}) \otimes \mathrm{L}^{1}(M)\right) p$ and commutes with $\psi(N)$. Hence, $T T^{*}$ is affiliated with $A$ and in particular, bounded. Hence, $T \in \mathcal{L}$.

So, we have to prove that $\mathcal{L}$ is finite dimensional. Let $p_{1}, \ldots, p_{r}$ be a maximal set of mutually orthogonal minimal projections in $A$ for which there exist $v_{i} \in \mathcal{L}$ with $v_{i} v_{i}^{*}=p_{i}$. For every $i$, let 
$q_{i 1}, \ldots, q_{i s_{i}}$ be a maximal set of mutually orthogonal projections in $q M^{m} q$ for which there exist $v_{i j} \in \mathcal{L}$ satisfying $v_{i j} v_{i j}^{*}=p_{i}$ and $v_{i j}^{*} v_{i j}=q_{i j}$. Since $\operatorname{Tr}\left(q_{i j}\right)=\operatorname{Tr}\left(p_{i}\right)$ for every $j$, it follows that $s_{i}<\infty$. It is now easy to check that

$$
\mathcal{L}=\operatorname{span}\left\{v_{i j} \mid i=1, \ldots, r, j=1, \ldots, s_{i}\right\} .
$$

\subsection{Connes tensor product versus product in a given module}

For the convenience of the reader, we prove the following elementary and probably well known lemma. It will be used several times in this paper.

Lemma 2.2. Let $N \subset M$ be an irreducible inclusion of $I I_{1}$ factors. Suppose that $\mathcal{K} \subset \mathrm{L}^{2}(M)$ is an $N$-N-subbimodule of finite index.

1. Choose a projection $p \in N^{n}$, a finite index inclusion $\varphi: N \rightarrow p N^{n} p$ and an $N$ - $N$-bimodular unitary

$$
U:{ }_{\varphi(N)} p\left(\mathbb{C}^{n} \otimes \mathrm{L}^{2}(N)\right)_{N} \rightarrow{ }_{N} \mathcal{K}_{N}
$$

Then, $U\left(p\left(\mathbb{C}^{n} \otimes N\right)\right)=\mathcal{K} \cap M$ and defining $v \in\left(\mathbb{C}^{n}\right)^{*} \otimes M$ by the formula

$$
v:=\sum_{i=1}^{n} e_{i}^{*} \otimes v_{i} \quad \text { with } \quad v_{i}:=U\left(p\left(e_{i} \otimes 1\right)\right),
$$

we have $v p=v$, av $=v \varphi(a)$ for all $a \in N$ and $U(\xi)=v \xi$ for all $\xi \in p\left(\mathbb{C}^{n} \otimes \mathrm{L}^{2}(N)\right)$. In particular, $\mathcal{K} \cap M=\operatorname{span}\left\{v_{i} N \mid i=1, \ldots, n\right\}$ and $\mathcal{K} \cap M$ is dense in $\mathcal{K}$.

2. Let $P$ be a $I I_{1}$ factor and ${ }_{M} \mathcal{H}_{P}$ an $M$-P-bimodule. Suppose that $\mathcal{L} \subset \mathcal{H}$ is a closed $N-P$ subbimodule. Denote by $\mathcal{K} * \mathcal{L}$ the closure of $(\mathcal{K} \cap M) \mathcal{L}$ inside $\mathcal{H}$. Then, $\mathcal{K} * \mathcal{L}$ is an $N-P$ bimodule that is isomorphic to a subbimodule of $\mathcal{K} \otimes_{N} \mathcal{L}$. Furthermore, whenever $\mathcal{K}_{0} \subset \mathcal{K} \cap M$ is such that $\mathcal{K}_{0} \subset \mathcal{K}$ is dense, also $\mathcal{K}_{0} \mathcal{L}$ follows dense in $\mathcal{K} * \mathcal{L}$. If $\mathcal{K} * \mathcal{L}$ is non-zero and $\mathcal{K} \otimes_{N} \mathcal{L}$ is irreducible, it follows that $\mathcal{K} * \mathcal{L}$ and $\mathcal{K} \otimes_{N} \mathcal{L}$ are isomorphic $N$-P-bimodules.

By symmetry, similar statements hold on the right. In particular, whenever ${ }_{P} \mathcal{H}_{M}$ is a $P-M-$ bimodule with closed $P-N$-subbimodule $\mathcal{L}$, we define $\mathcal{L} * \mathcal{K}$ as the closure of $\mathcal{L}(\mathcal{K} \cap M)$ inside $\mathcal{H}$ and find that $\mathcal{L} * \mathcal{K}$ is isomorphic with a $P$-N-subbimodule of $\mathcal{L} \otimes_{N} \mathcal{K}$.

Proof. Choose a projection $p \in N^{n}$, a finite index inclusion $\varphi: N \rightarrow p N^{n} p$ and an $N$ - $N$-bimodular unitary

$$
U:{ }_{\varphi(N)} p\left(\mathbb{C}^{n} \otimes \mathrm{L}^{2}(N)\right)_{N} \rightarrow{ }_{N} \mathcal{K}_{N}
$$

Define $v_{i} \in \mathrm{L}^{2}(M)$ by the formula $v_{i}:=U\left(p\left(e_{i} \otimes 1\right)\right)$. Put $v:=\sum_{i=1}^{n} e_{i}^{*} \otimes v_{i}$, which belongs to $\left(\mathbb{C}^{n}\right)^{*} \otimes \mathrm{L}^{2}(M)$. By construction, $\operatorname{span}\left\{v_{i} N \mid i=1, \ldots, n\right\}$ is dense in $\mathcal{K}$ and $U(\xi)=v \xi$ for all $\xi \in p\left(\mathbb{C}^{n} \otimes N\right)$. Since $U$ is $N$-N-bimodular, we have $a v=v \varphi(a)$ for all $a \in N$ and, in particular, $v=v p$. It follows that $v v^{*}$ is an element of $\mathrm{L}^{1}(M)$ commuting with $N$. By the irreducibility of $N \subset M$, we get $v v^{*} \in \mathbb{C} 1$. In particular, $v$ is bounded and $v_{i} \in M$ for every $i$.

Since $U(\xi)=v \xi$ for all $\xi \in p\left(\mathbb{C}^{n} \otimes N\right)$, also $U^{*}(b)=\left(\right.$ id $\left.\otimes E_{N}\right)\left(v^{*} b\right)$ for all $b \in M$. In particular, $U^{*}(M) \subset p\left(\mathbb{C}^{n} \otimes N\right)$ and it follows that $\mathcal{K} \cap M=U\left(p\left(\mathbb{C}^{n} \otimes N\right)\right)=\operatorname{span}\left\{v_{i} N \mid i=1, \ldots, n\right\}$. This proves the first part of the lemma. 
To prove the second part, let $P$ be a $\mathrm{II}_{1}$ factor and ${ }_{M} \mathcal{H}_{P}$ an $M$ - $P$-bimodule with closed $N$ - $P$ subbimodule $\mathcal{L}$. Define $\mathcal{K} * \mathcal{L}$ as the closure of $(\mathcal{K} \cap M) \mathcal{L}$ inside $\mathcal{H}$. Define the subspace $\mathcal{L}_{0} \subset \mathcal{L}$ of vectors $\xi \in \mathcal{L}$ such that the map $N \rightarrow \mathcal{L}: a \mapsto a \xi$ extends to a bounded operator from $\mathrm{L}^{2}(N)$ to $\mathcal{L}$. Then, $\mathcal{L}_{0}$ is dense in $\mathcal{L}$. Fix $\xi \in \mathcal{L}_{0}$. We claim that the map $R: \mathcal{K} \cap M \rightarrow \mathcal{H}: a \mapsto a \xi$ extends to a bounded operator from $\mathcal{K}$ to $\mathcal{H}$. Since $\xi \in \mathcal{L}_{0}$, the map

$$
S: \mathbb{C}^{n} \otimes N \rightarrow \mathcal{H}: e_{i} \otimes a \mapsto v_{i} a \xi
$$

extends to a bounded operator from $\mathbb{C}^{n} \otimes \mathrm{L}^{2}(N)$ to $\mathcal{H}$, that we still denote by $S$. By construction, $S(\varphi(a) \eta)=a S(\eta)$ for all $a \in N$ and $\eta \in \mathbb{C}^{n} \otimes \mathrm{L}^{2}(N)$. In particular, $S(\eta)=S(p \eta)$ for all $\eta \in \mathbb{C}^{n} \otimes \mathrm{L}^{2}(N)$. It follows that $R(U(\eta))=S(\eta)$ for all $\eta \in p\left(\mathbb{C}^{n} \otimes N\right)$. Since $U$ is unitary and $\mathcal{K} \cap M$ equals $U\left(p\left(\mathbb{C}^{n} \otimes N\right)\right)$, the claim is proven.

Suppose now that $\mathcal{K}_{0} \subset \mathcal{K} \cap M$ and that $\mathcal{K}_{0} \subset \mathcal{K}$ is dense. From the claim in the previous paragraph, we get

$$
\mathcal{K} * \mathcal{L}=\overline{(\mathcal{K} \cap M) \mathcal{L}}=\overline{(\mathcal{K} \cap M) \mathcal{L}_{0}}=\overline{\mathcal{K}_{0} \mathcal{L}_{0}}=\overline{\mathcal{K}_{0} \mathcal{L}} .
$$

So, $\mathcal{K}_{0} \mathcal{L}$ is dense in $\mathcal{K} * \mathcal{L}$.

The Connes tensor product $\mathcal{K} \otimes_{N} \mathcal{L}$ can be realized as the $N$-P-bimodule ${ }_{\varphi(N)} p\left(\mathbb{C}^{n} \otimes \mathcal{L}\right)_{P}$. The linear operator $T: p\left(\mathbb{C}^{n} \otimes \mathcal{L}\right) \rightarrow \mathcal{H}: T(\xi)=v \xi$ is $N$-P-bimodular with range $\operatorname{span}\left\{v_{i} \mathcal{L} \mid i=1, \ldots, n\right\}$. From the results above, it follows that the closure of the range of $T$ equals $\mathcal{K} * \mathcal{L}$. Taking the polar decomposition of $T$, we find an $N$-P-bimodular isometry of $\mathcal{K} * \mathcal{L}$ into $\mathcal{K} \otimes_{N} \mathcal{L}$.

\subsection{Quasi-normalizers}

Let $(M, \tau)$ be a tracial von Neumann algebra and $N \subset M$ a von Neumann subalgebra.

- The quasi-normalizer of $N$ inside $M$ is defined as:

$$
\mathrm{QN}_{M}(N)=\left\{a \in M \mid \exists a_{1}, \ldots, a_{n}, b_{1}, \ldots, b_{m} \in M \text { s.t. } N a \subset \sum_{i=1}^{n} a_{i} N \text { and } a N \subset \sum_{i=1}^{m} N b_{i}\right\} .
$$

- The inclusion $N \subset M$ is called quasi-regular if $\mathrm{QN}_{M}(N)^{\prime \prime}=M$.

Remark that the quasi-normalizer of $N \subset M$ is a unital $*$-subalgebra of $M$ containing $N$.

Let $\Gamma$ be a group and $\Lambda \subset \Gamma$ a subgroup.

- The commensurator of $\Lambda \subset \Gamma$ is defined as

$$
\operatorname{Comm}_{\Gamma}(\Lambda):=\left\{g \in \Gamma \mid g \Lambda g^{-1} \cap \Lambda \text { has finite index in } g \Lambda g^{-1} \text { and in } \Lambda\right\} .
$$

- The inclusion $\Lambda \subset \Gamma$ is called almost normal if $\operatorname{Comm}_{\Gamma}(\Lambda)=\Gamma$.

Remark that the inclusion $\mathrm{L}(\Lambda) \subset \mathrm{L}(\Gamma)$ is quasi-regular if and only if the inclusion $\Lambda \subset \Gamma$ is almost normal. A typical example of an almost normal subgroup is $\operatorname{SL}(n, \mathbb{Z}) \subset \operatorname{SL}(n, \mathbb{Q})$.

Again we prove an elementary lemma for the convenience of the reader. 
Lemma 2.3. Let $N \subset M$ be an irreducible, quasi-regular inclusion of $I I_{1}$ factors. Then, ${ }_{N} \mathrm{~L}^{2}(M)_{N}$ is the orthogonal direct sum of a family of irreducible, finite index $N$ - $N$-subbimodules $K_{i} \subset \mathrm{L}^{2}(M)$, $i \in I$. Writing $K_{i}^{0}:=K_{i} \cap M$, we have, for any choice of decomposition, $\operatorname{span}\left\{K_{i}^{0} \mid i \in I\right\}=$ $\mathrm{QN}_{M}(N)$.

Proof. Whenever $a \in \mathrm{QN}_{M}(N)$, the closure of $N a N$ inside $\mathrm{L}^{2}(M)$ is an $N$ - $N$-subbimodule of finite index. So, the linear span of all finite index $N$ - $N$-subbimodules of $\mathrm{L}^{2}(M)$, is dense in $\mathrm{L}^{2}(M)$. Hence, $\mathrm{L}^{2}(M)$ can be decomposed into an orthogonal direct sum of a family of irreducible, finite index $N$ - $N$-subbimodules $K_{i} \subset \mathrm{L}^{2}(M), i \in I$. Write $K_{i}^{0}:=K_{i} \cap M$. By Lemma 2.2 and its right-handed analogue, we find $v_{j}^{i}, j=1, \ldots, n_{i}$ and $w_{j}^{i}, j=1, \ldots, m_{i}$ in $K_{i}^{0}$ such that

$$
\operatorname{span}\left\{v_{j}^{i} N \mid j=1, \ldots, n_{i}\right\}=K_{i}^{0}=\operatorname{span}\left\{N w_{j}^{i} \mid j=1, \ldots, m_{i}\right\} .
$$

Since $N K_{i}^{0} N=K_{i}^{0}$, it follows that $K_{i}^{0} \subset \mathrm{QN}_{M}(N)$.

The proof of Lemma 2.2 yields $v_{i} \in\left(\mathbb{C}^{n_{i}}\right)^{*} \otimes M$ such that the orthogonal projection $P_{i}$ of $\mathrm{L}^{2}(M)$ onto $K_{i}$ satisfies $P_{i}(a)=v_{i}\left(\mathrm{id} \otimes E_{N}\right)\left(v_{i}^{*} a\right)$ for all $a \in M$. In particular, $P_{i}(M)=K_{i}^{0}$.

Let now $a \in \mathrm{QN}_{M}(N)$ and decompose the closure of $N a N$ inside $\mathrm{L}^{2}(M)$ as a direct sum of irreducible $N$ - $N$-subbimodules $H_{1}, \ldots, H_{n}$. Lemma 2.1 implies that for every $i=1, \ldots, n$, there exists a finite subset $I_{i} \subset I$ such that $H_{i} \approx K_{j}$ whenever $j \in I \backslash I_{i}$. So, we find a finite subset $I_{0} \subset I$ such that $N a N \subset \operatorname{span}\left\{K_{i} \mid i \in I_{0}\right\}$. But then,

$$
a=\sum_{i \in I_{0}} P_{i}(a) \in \operatorname{span}\left\{K_{i}^{0} \mid i \in I\right\} .
$$

\subsection{Amalgamated free products}

We recall now some basic facts and notations about amalgamated free products, see [14] and [23] for more details. Let $\left(M_{0}, \tau_{0}\right)$ and $\left(M_{1}, \tau_{1}\right)$ be tracial von Neumann algebras with a common von Neumann subalgebra $N$ such that $\tau_{0 \mid N}=\tau_{1 \mid N}$. We denote by $E_{i}$ the unique trace preserving conditional expectation of $M_{i}$ onto $N$. The amalgamated free product $M_{0} *_{N} M_{1}$ is, up to $E$ preserving isomorphism, the unique pair $(M, E)$ satisfying the following two conditions.

- The von Neumann algebra $M$ is generated by embeddings of $M_{0}$ and $M_{1}$ that are identical on $N$, and is equipped with a conditional expectation $E: M \rightarrow N$.

- The subalgebras $M_{0}$ and $M_{1}$ are free with amalgamation over $N$ with respect to $E$. This means that $E\left(x_{1} \cdots x_{n}\right)=0$ whenever $x_{j} \in M_{i_{j}}$ such that $E_{i_{j}}\left(x_{j}\right)=0$ and $i_{1} \neq i_{2}$, $i_{2} \neq i_{3}, \ldots, i_{n-1} \neq i_{n}$.

The amalgamated free product $M_{0} *_{N} M_{1}$ has a dense $*$-subalgebra given by

$$
N \oplus \bigoplus_{n \geq 1}\left(\bigoplus_{i_{1} \neq i_{2}, \ldots, i_{n-1} \neq i_{n}} \stackrel{\circ}{M_{i_{1}}} \cdots{\stackrel{\circ}{i_{n}}}_{i_{n}}\right),
$$

where $\stackrel{\circ}{M}_{i_{k}}:=M_{i_{k}} \ominus N$. The von Neumann algebra $M_{0} *_{N} M_{1}$ has a trace, defined by $\tau:=\tau_{0} \circ E=$ $\tau_{1} \circ E$. 


\subsection{Minimal actions of compact groups and bimodule categories}

We assume all compact groups to be second countable. A strongly continuous action $G \curvearrowright M$ of a compact group $G$ on a $\mathrm{II}_{1}$ factor $M$ is said to be minimal if the map $G \rightarrow \operatorname{Aut}(M)$ is injective and if $M \cap\left(M^{G}\right)^{\prime}=\mathbb{C} 1$. Here, $M^{G}$ is the von Neumann algebra of $G$-fixed points in $M$. We always denote by $H_{\pi}$ the Hilbert space of the representation $\pi$ and by $\epsilon$ the trivial representation.

Every minimal action $G \curvearrowright M$ gives rise to a tensor functor $\operatorname{Rep}(G) \rightarrow \operatorname{Bimod}\left(M^{G}\right)$. This goes back to [19] and can be stated explicitly as follows.

Proposition 2.4. Let $G$ be a second countable compact group and $\sigma: G \curvearrowright M$ a minimal action. Set $P:=M^{G}$. Then,

$$
\begin{aligned}
& \operatorname{Rep}(G) \rightarrow \operatorname{Bimod}(P): \pi \mapsto{ }_{P} \operatorname{Mor}\left(H_{\pi}, \mathrm{L}^{2}(M)\right)_{P} \quad \text { where } \quad\langle S, T\rangle:=\operatorname{Tr}\left(S^{*} T\right) \\
& \text { and }(a \cdot S \cdot b)(\xi)=a S(\xi) b \quad \text { for all } S, T \in \operatorname{Mor}\left(H_{\pi}, \mathrm{L}^{2}(M)\right), a, b \in P, \xi \in H_{\pi}
\end{aligned}
$$

defines a fully faithful tensor functor from the category $\operatorname{Rep}(G)$ of finite dimensional unitary representation of $G$ to the category $\operatorname{Bimod}(P)$ of finite index P-P-bimodules.

Let $\sigma: G \curvearrowright M$ be a minimal action and choose a complete set $\operatorname{Irr}(G)$ of inequivalent, irreducible unitary representations of $G$. For every $\pi \in \operatorname{Irr}(G)$, we choose and fix a unitary $V_{\pi} \in \mathrm{B}\left(H_{\pi}\right) \otimes M$ satisfying $\left(\right.$ id $\left.\otimes \sigma_{g}\right)\left(V_{\pi}\right)=V_{\pi}(\pi(g) \otimes 1)$, see e.g. [24, Theorem 12 and following comments].

Put $P:=M^{G}$. For every $\pi \in \operatorname{Irr}(G)$, the map

$$
\psi_{\pi}: P \rightarrow \mathrm{B}\left(H_{\pi}\right) \otimes P: \psi_{\pi}(a)=V_{\pi}(1 \otimes a) V_{\pi}^{*}
$$

defines an irreducible, finite index inclusion. Define the Hilbert space $H\left(\psi_{\pi}\right)=H_{\pi}^{*} \otimes \mathrm{L}^{2}(P)$, which is a $P$-P-bimodule as ${ }_{P}\left(H_{\pi}^{*} \otimes \mathrm{L}^{2}(P)\right)_{\psi_{\pi}(P)}$. Of course, in the light of Proposition 2.4, we have $H\left(\psi_{\pi}\right) \cong \operatorname{Mor}\left(H_{\pi}, \mathrm{L}^{2}(M)\right)$ as $P$-P-bimodules.

We introduce now some notations concerning spectral subspaces of irreducible representations. Denote by $\operatorname{Mor}(\pi, M)$ the space of linear maps $S: H_{\pi} \rightarrow M$ satisfying $\sigma_{g} \circ S=S \circ \pi(g)$. We denote the linear span of $\operatorname{Mor}(\pi, M) H_{\pi}$ as $L^{0}(\pi) \subset M$. The closure of $L^{0}(\pi)$ inside $\mathrm{L}^{2}(M)$ is denoted by $L(\pi)$.

- As a $P$-P-bimodule, $L(\pi)$ is the direct sum of $\operatorname{dim}(\pi)$ copies of $H\left(\psi_{\pi}\right)$. More precisely, if you consider on $\mathrm{B}\left(H_{\pi}\right) \otimes P$ the scalar product given by $\operatorname{Tr} \otimes \tau$, the map

$$
\theta_{\pi}: 1 \otimes P\left(\mathrm{~B}\left(H_{\pi}\right) \otimes P\right)_{\psi_{\pi}(P)} \rightarrow{ }_{P} L^{0}(\pi)_{P}: \theta_{\pi}(a)=\operatorname{dim}(\pi)^{1 / 2}(\operatorname{Tr} \otimes \mathrm{id})\left(a V_{\pi}\right)
$$

is $P$-P-bimodular, bijective and extends to an isometry $\mathrm{B}\left(H_{\pi}\right) \otimes \mathrm{L}^{2}(P) \hookrightarrow \mathrm{L}^{2}(M)$.

- The adjoint of $\theta_{\pi}$ is given by $E_{\pi}:=\theta_{\pi}^{*}$ satisfying

$$
E_{\pi}(b)=\operatorname{dim}(\pi)^{1 / 2} \int_{G}\left(\pi(g)^{*} \otimes \sigma_{g}(b)\right) V_{\pi}^{*} d g
$$

for all $b \in M$.

- Since every unitary representation of $G$ splits as a direct sum of irreducibles, we have

$$
\sum_{\pi \in \operatorname{Irr}(G)} E_{\pi}^{*} E_{\pi}=1
$$

Equivalently, $\mathrm{L}^{2}(M)$ is the orthogonal direct sum of the subspaces $L(\pi), \pi \in \operatorname{Irr}(G)$. 
Remark 2.5. The coefficients of the unitaries $V_{\pi}$ quasi-normalize $M^{G}$ and so, the inclusion $M^{G} \subset$ $M$ is quasi-regular. In fact, by Lemma 2.3, the quasi-normalizer of $M^{G}$ inside $M$ equals the linear span of all $L^{0}(\pi), \pi \in \operatorname{Irr}(G)$.

For later use (in the proof of Lemma 3.10), we record the following elementary property.

Lemma 2.6. Let $\sigma: G \curvearrowright M$ be a minimal action of a compact group $G$ on a $I I_{1}$ factor $M$. Let $\pi$ and $\eta$ be irreducible representations of $G$. Take $\mu_{1}, \ldots, \mu_{n} \in \operatorname{Irr}(G)$, with possible repetitions, and isometries $v_{i} \in \operatorname{Mor}\left(\mu_{i}, \pi \otimes \eta\right)$ satisfying $\sum_{i=1}^{n} v_{i} v_{i}^{*}=1$. There exist $X_{i} \in \mathrm{B}\left(H_{\mu_{i}}, H_{\pi} \otimes H_{\eta}\right) \otimes M^{G}$ with $X_{i}^{*} X_{i}=1$ for all $i$ and $\sum_{i=1}^{n} X_{i} X_{i}^{*}=1$ such that

$$
\left(V_{\pi}\right)_{13}\left(V_{\eta}\right)_{23}=\sum_{i=1}^{n} X_{i} V_{\mu_{i}}\left(v_{i}^{*} \otimes 1\right) .
$$

\subsection{Freeness and free products of fusion algebras}

The notions of freeness and free product of fusion algebras were introduced in [2, Section 1.2], in the study of free composition of subfactors. For convenience, we recall the definition.

Definition 2.7 ([2, Section 1.2]). Let $\mathcal{A}$ be a fusion algebra and $\mathcal{A}_{i} \subset \mathcal{A}$ fusion subalgebras for $i=1,2$. We say that $\mathcal{A}_{1}$ and $\mathcal{A}_{2}$ are free inside $\mathcal{A}$ if every alternating product of irreducibles in $\mathcal{A}_{i} \backslash\{e\}$, remains irreducible and different from $\{e\}$.

Given fusion algebras $\mathcal{A}_{1}$ and $\mathcal{A}_{2}$, there is up to isomorphism a unique fusion algebra $\mathcal{A}$ generated by copies of $\mathcal{A}_{1}$ and $\mathcal{A}_{2}$ that are free. We call this unique $\mathcal{A}$ the free product of $\mathcal{A}_{1}$ and $\mathcal{A}_{2}$ and denote it by $\mathcal{A}_{1} * \mathcal{A}_{2}$.

Denote by $R$ the hyperfinite $\mathrm{II}_{1}$ factor. The fusion algebra $\mathrm{FAlg}(R)$ is huge, in the sense that $\mathrm{FAl}(R)$ contains many free fusion subalgebras. More precisely, it was shown in Theorem 5.1 of [21] that countable fusion subalgebras of $\mathrm{FAlg}(R)$ can be made free by conjugating one of them with an automorphism of $R$ (see Theorem 2.8 below). Note that the same result has first been proven for countable subgroups of $\operatorname{Out}(R)$ in [7]. In both cases, the key ingredients come from [13].

Let $M$ be a $\mathrm{II}_{1}$ factor and ${ }_{M} \mathcal{K}_{M}$ a finite index $M$ - $M$-bimodule. Whenever $\alpha \in \operatorname{Aut}(M)$, we define the conjugation of $K$ by $\alpha$ as the bimodule $\mathcal{K}^{\alpha}:=H\left(\alpha^{-1}\right) \otimes_{M} \mathcal{K} \otimes_{M} H(\alpha)$.

Theorem 2.8 (Theorem 5.1 in [21]). Let $R$ be the hyperfinite $I I_{1}$ factor and $\mathcal{A}_{0}, \mathcal{A}_{1}$ two countable fusion subalgebras of $\mathrm{FAlg}(R)$. Then,

$$
\left\{\alpha \in \operatorname{Aut}(R) \mid \mathcal{A}_{0}^{\alpha} \text { and } \mathcal{A}_{1} \text { are free }\right\}
$$

is $a G_{\delta}$-dense subset of $\operatorname{Aut}(R)$.

\subsection{Intertwining by bimodules}

In this section, we briefly recall Popa's intertwining-by-bimodules technique introduced in [12]. This very powerful technique is used to deduce unitary conjugacy of two von Neumann subalgebras $A$ and $B$ of a tracial von Neumann algebra $(M, \tau)$ from their weak containment $A \prec_{M} B$ that we define now. 
Definition 2.9. Let $(M, \tau)$ be a von Neumann algebra with normal faithful tracial state. Let $A, B \subset M^{n}$ be possibly non-unital embeddings. We write $A \underset{M}{\prec} B$ if $1_{A}\left(\mathrm{M}_{n}(\mathbb{C}) \otimes \mathrm{L}^{2}(M)\right) 1_{B}$ contains a non-zero $A$ - $B$-subbimodule $K$ with $\operatorname{dim}\left(K_{B}\right)<\infty$.

By [10, Theorem 2.1] (see also Appendix $\mathrm{C}$ in [22]), we have $A \prec_{M} B$ if and only if there exist $m \in \mathbb{N}$, a non-zero partial isometry $v \in 1_{A}\left(\mathbb{C}^{n}\left(\mathbb{C}^{m} \otimes \mathbb{C}^{n}\right)^{*} \otimes M\right)\left(1 \otimes 1_{B}\right)$ and a possibly nonunital $*$-homomorphism $\psi: A \rightarrow \mathrm{M}_{m}(\mathbb{C}) \otimes B$ satisfying $a v=v \psi(a)$ for all $a \in A$. In particular, writing for $i=1, \ldots, m, v_{i}:=v\left(e_{i} \otimes 1 \otimes 1\right)$, we have found $v_{1}, \ldots, v_{m} \in 1_{A} M^{n} 1_{B}$ such that $\operatorname{span}\left\{v_{i} B \mid i=1, \ldots, m\right\}$ is a left $A$-module.

\subsection{Property (T) for $\mathrm{II}_{1}$ factors}

Property $(\mathrm{T})$ for finite von Neumann algebras was defined by Connes and Jones in [4]: a $\mathrm{II}_{1}$ factor $(M, \tau)$ has property (T) if and only if there exists $\epsilon>0$ and a finite subset $F \subset M$ such that every $M$ - $M$-bimodule $H$ that has a unit vector $\xi$ satisfying $\|x \xi-\xi x\| \leq \epsilon$, for all $x \in F$, actually has a non-zero $M$-central vector $\xi_{0}$, meaning that $x \xi_{0}=\xi_{0} x$, for all $x \in M$. Note that an ICC group $\Gamma$ has property $(\mathrm{T})$ if and only if the $\mathrm{II}_{1}$ factor $\mathrm{L}(\Gamma)$ has property $(\mathrm{T})$ in the sense of Connes and Jones.

\subsection{Rigid subalgebras of amalgamated free products}

For the convenience of the reader we quote some of the results obtained by Ioana, Peterson and Popa in [7, Theorems 1.1 and 5.1]. We only state the version as needed in this paper and give a few comments.

Theorem 2.10 (Theorems 1.1 and 5.1 in [7]). Let $\left(M_{0}, \tau_{0}\right)$ and $\left(M_{1}, \tau_{1}\right)$ be von Neumann algebras with faithful normal tracial states. Let $N$ be a common von Neumann subalgebra of $M_{0}$ and $M_{1}$ with $\left.\tau_{0}\right|_{N}=\left.\tau_{1}\right|_{N}$. Define the amalgamated free product $M:=M_{0} *_{N} M_{1}$ with respect to the unique trace-preserving conditional expectations.

1. Let $p \in M_{0}^{n}$ be a projection and $Q \subset p M_{0}^{n} p$ a von Neumann subalgebra satisfying $Q \nprec_{M_{0}} N$. Whenever $\mathcal{K} \subset p\left(\mathbb{C}^{n} \otimes \mathrm{L}^{2}(M)\right)$ is a $Q$ - $M_{0}$-subbimodule with $\operatorname{dim}\left(\mathcal{K}_{M_{0}}\right)<\infty$, we have $\mathcal{K} \subset$ $p\left(\mathbb{C}^{n} \otimes \mathrm{L}^{2}\left(M_{0}\right)\right)$. In particular, the quasi-normalizer of $Q$ inside $p M^{n} p$ is contained in $p M_{0}^{n} p$.

2. Assume that $M_{0}$ is a factor. Let $p \in M^{n}$ be a projection and $Q \subset p M^{n} p$ a von Neumann subalgebra with the relative property (T) in the sense of Popa [12, Definition 4.2] (which holds, in particular, if $Q$ is a $I I_{1}$ factor having the property $(T)$ of Connes and Jones explained in Section 2.10). Assume that $Q \nprec_{M} M_{1}$. Then, there exists $u \in M^{n}$ satisfying $u u^{*}=p$ and $u^{*} Q u \subset M_{0}^{n}$.

The first item is precisely [7, Theorem 1.1]. Note, in comparison with [7], that working with matrices over $M$ is not really more general, because we identify $M^{n}$ with $M_{0}^{n} *_{N^{n}} M_{1}^{n}$.

The second item is a special case of [7, Theorem 5.1]. Again, it is not a real generalization to work with matrices over $M$, so that we may assume $n=1$. Next, since $M$ is a factor, we can choose a von Neumann subalgebra $\tilde{Q} \subset M$, containing $p$, such that $Q=p \tilde{Q} p$ and such that the inclusion $\tilde{Q} \subset M$ still has the relative property $(\mathrm{T})$ and still satisfies $\tilde{Q} \nprec_{M} M_{1}$. Then, [7, Theorem 5.1] provides a unitary $v \in M$ such that $v^{*} \tilde{Q} v \subset M_{0}$. We put $u=p v$. Finally, in [7, Theorem 5.1], the extra assumption is made that the inclusions $N \subset M_{i}$ are homogeneous. But, as explained in [6, Section 5.2], the homogeneity assumption is superfluous. 


\section{Proof of Theorem 1.1}

Fix a second countable compact group $G$ and an action $G \stackrel{\sigma}{\curvearrowright} M_{1}$ on the $\mathrm{II}_{1}$ factor $M_{1}$. Denote $N=M_{1}^{G}$ and fix an inclusion $N \subset M_{0}$ into the $\mathrm{II}_{1}$ factor $M_{0}$. We are interested in the $\mathrm{II}_{1}$ factor

$$
M:=M_{0} *_{N} M_{1}
$$

and extend the action $G \curvearrowright M_{1}$ to an action $G \curvearrowright M$ by acting trivially on $M_{0}$.

\section{Assumptions}

1. Assumption on the action $G \stackrel{\sigma}{\curvearrowright} M_{1}: \sigma$ is minimal and $M_{1}$ is hyperfinite.

2. Assumptions on the inclusion $N \subset M_{0}$.

2.a) The inclusion $N \subset M_{0}$ is irreducible, i.e. $N^{\prime} \cap M_{0}=\mathbb{C} 1$ and is quasi-regular (see Section $2.5)$.

2.b) A condition on absence of finite dimensional unitary representations (cf. Lemmas 3.2 and 3.3). Denote by $\mathcal{F}_{0}$ the fusion subalgebra of $\mathrm{FAlg}(N)$ generated by the finite index $N$ - $N$-subbimodules of ${ }_{N} \mathrm{~L}^{2}\left(M_{0}\right)_{N}$. Whenever ${ }_{M_{0}} \mathcal{K}_{M_{0}}$ is an irreducible finite index $M_{0^{-}} M_{0^{-}}$ bimodule containing a non-zero element of $\mathcal{F}_{0}$ as $N$-N-subbimodule, we have ${ }_{M_{0}} \mathcal{K}_{M_{0}} \cong$ $M_{0} \mathrm{~L}^{2}\left(M_{0}\right)_{M_{0}}$.

3. Rigidity assumption: there exists $N \subset N_{0} \subset M_{0}$ such that $N_{0}$ has property (T) in the sense of Connes and Jones [4] and such that $N_{0} \subset M_{0}$ is quasi-regular.

4. Relation between $G \stackrel{\sigma}{\curvearrowright} M_{1}$ and $N \subset M_{0}$. Denote by $\mathcal{F}$ the fusion subalgebra of $\operatorname{FAlg}(N)$ generated by the finite index $N$-N-bimodules that arise as $N$-N-subbimodule of a finite index $M_{0}-M_{0}$-bimodule. Then, $\mathcal{F}$ is free with respect to the canonical image of $\operatorname{Rep}(G)$ in $\operatorname{FAlg}(N)$, given by the minimal action $\sigma$ (see Proposition 2.4 and recall that $N=M_{1}^{G}$ ).

Remark 3.1. If $N \subset M_{0}$ is an irreducible inclusion of $\mathrm{II}_{1}$ factors having the relative property (T) (so, in particular, if $N \subset M_{0}$ satisfies assumption 3), one can repeat the proof of [21, Lemma 4.1] and it follows that the fusion algebra $\mathcal{F}$ defined in assumption 4 is countable.

We now clarify the slightly mysterious assumption 2.b. The first of the following two lemmas is not used in the paper, but makes the link with absence of finite dimensional unitary representations.

Lemma 3.2. Let $N$ be a $I I_{1}$ factor and $\Gamma \curvearrowright N$ an action of the countable group $\Gamma$ by outer automorphisms of $N$. Put $M_{0}:=N \rtimes \Gamma$. Then, the inclusion $N \subset M_{0}$ satisfies assumption 2.b if and only if $\Gamma$ has no non-trivial finite dimensional unitary representations.

Proof. Since $\Gamma \curvearrowright N$ is outer, the inclusion $N \subset M_{0}$ is irreducible. Denote by $\left(u_{g}\right)_{g \in \Gamma}$ the canonical unitaries in $M_{0}$. Since every $a u_{g}, g \in \Gamma, a \in \mathcal{U}(N)$, normalizes $N$, the inclusion $N \subset M_{0}$ is regular. If $\pi: \Gamma \rightarrow \mathcal{U}(H)$ is a non-trivial irreducible finite dimensional unitary representation, define $\mathcal{K}=$ $H \otimes \mathrm{L}^{2}\left(M_{0}\right)$ with bimodule action given by

$$
\left(a u_{g}\right) \cdot \xi=\left(\pi(g) \otimes a u_{g}\right) \xi \quad \text { and } \quad \xi \cdot b=\xi b \quad \text { for all } a \in N, g \in \Gamma, b \in M_{0} .
$$


Then, $M_{0} \mathcal{K}_{M_{0}}$ is an irreducible finite index $M_{0}-M_{0}$-bimodule and ${ }_{M_{0}} \mathcal{K}_{M_{0}} \approx{ }_{M_{0}} \mathrm{~L}^{2}\left(M_{0}\right)_{M_{0}}$. Nevertheless, we have the $N-N$-subbimodule $H \otimes \mathrm{L}^{2}(N) \subset \mathcal{K}$, which is a $\operatorname{sum}$ of $\operatorname{dim}(H)$ copies of the trivial $N$-N-bimodule and hence, belongs to $\mathcal{F}_{0}$. So, assumption 2.b fails.

Conversely, suppose that $\Gamma$ has no non-trivial finite dimensional unitary representations and denote the action by $\Gamma \stackrel{\rho}{\curvearrowright} N$. Observe that the irreducible elements in $\mathcal{F}_{0}$ are precisely the $N$ - $N$-bimodules $H\left(\rho_{s}\right), s \in \Gamma$, defined on the Hilbert space $\mathrm{L}^{2}(N)$ with bimodule action

$$
a \cdot \xi \cdot b:=a \xi \rho_{s}(b) \text { for all } \xi \in \mathrm{L}^{2}(N), a, b \in N .
$$

Let $M_{0} \mathcal{K}_{M_{0}}$ be an irreducible finite index $M_{0}-M_{0}$-bimodule. Let $\mathcal{H} \subset \mathcal{K}$ be an $N$ - $N$-subbimodule such that ${ }_{N} \mathcal{H}_{N} \cong{ }_{N} H\left(\rho_{s}\right)_{N}$ for a certain $s \in \Gamma$. By irreducibility of $\mathcal{K}$, it follows that the span of all $u_{r} \cdot \mathcal{H} \cdot u_{k}, r, k \in \Gamma$, is dense in $\mathcal{K}$. So, $\mathcal{K}$ decomposes as a direct sum of $N$ - $N$-subbimodules, each of them being isomorphic to one of the $H\left(\rho_{r}\right), r \in \Gamma$. Multiplying on the right by $u_{r}$, it follows that every $N$ - $M_{0}$-subbimodule of $\mathcal{K}$ contains the trivial $N$ - $N$-bimodule as an $N$ - $N$-subbimodule.

Write ${ }_{M_{0}} \mathcal{K}_{M_{0}} \cong \psi\left(M_{0}\right) p\left(\mathbb{C}^{n} \otimes \mathrm{L}^{2}\left(M_{0}\right)\right)_{M_{0}}$. Since $\mathcal{K}$ has finite index and $N \subset M_{0}$ is irreducible, the relative commutant $A:=\psi(N)^{\prime} \cap p M_{0}^{n} p$ is finite dimensional. By the previous paragraph, we find for every minimal projection $q \in A$, a non-zero vector $\xi \in q\left(\mathbb{C}^{n} \otimes \mathrm{L}^{2}\left(M_{0}\right)\right)$ satisfying $\psi(a) \xi=\xi a$ for all $a \in N$. As an element of $q\left(\mathrm{M}_{n}(\mathbb{C}) \otimes \mathrm{L}^{1}\left(M_{0}\right)\right) q$, the operator $\xi \xi^{*}$ commutes with $\psi(N)$ and hence, is a multiple of $q$. So, we may assume that $\xi \in q\left(\mathbb{C}^{n} \otimes M_{0}\right)$ and $\xi \xi^{*}=q$. Since $N \subset M_{0}$ is irreducible, $\xi^{*} \xi=1$. Repeating this procedure for a family of minimal projections in $A$ summing to 1 , we find a unitary $X \in q\left(\mathbb{C}^{n}\left(\mathbb{C}^{m}\right)^{*} \otimes M_{0}\right)$ such that $X^{*} \psi(a) X=1 \otimes a$ for all $a \in N$.

So, we may assume that $p=1$ and $\psi(a)=1 \otimes a$ for all $a \in N$. But then, $\psi\left(u_{g}\right)=\pi(g) \otimes u_{g}$ for all $g \in \Gamma$. Since $\Gamma$ has no finite dimensional unitary representations, it follows that $\pi(g)=1$. By irreducibility of $\mathcal{K}$, it follows that $n=1$ and ${ }_{M_{0}} \mathcal{K}_{M_{0}} \cong{ }_{M_{0}} \mathrm{~L}^{2}\left(M_{0}\right)_{M_{0}}$.

Lemma 3.3. Let $\Gamma$ be a countable group and $\Lambda<\Gamma$ an almost normal subgroup. Let $\Omega \in \mathrm{Z}^{2}\left(\Gamma, S^{1}\right)$ be a scalar 2-cocycle. Put $N:=\mathrm{L}_{\Omega}(\Lambda)$ and $M_{0}:=\mathrm{L}_{\Omega}(\Gamma)$. If the following conditions hold, the inclusion $N \subset M_{0}$ satisfies assumption 2.b.

- For all finite index subgroups $\Lambda_{0}<\Lambda$, we have $\mathrm{L}_{\Omega}\left(\Lambda_{0}\right)^{\prime} \cap \mathrm{L}_{\Omega}(\Gamma)=\mathbb{C} 1$.

- The group $\Gamma$ has no non-trivial finite dimensional unitary representations.

Proof. Define the fusion subalgebra $\mathcal{F}_{0}$ of $\operatorname{FAlg}(N)$ as in assumption 2.b. We first claim that every ${ }_{N} \mathcal{H}_{N}$ in $\mathcal{F}_{0}$ is of good form, by which we mean that there exists $n$, a finite index inclusion $\gamma: N \rightarrow N^{n}$, elements $g_{1}, \ldots, g_{n} \in \Gamma$ and a finite index subgroup $\Lambda_{0}<\Lambda \cap \bigcap_{i=1}^{n} g_{i} \Lambda g_{i}^{-1}$ such that ${ }_{N} \mathcal{H}_{N} \cong \gamma(N)\left(\mathbb{C}^{n} \otimes \mathrm{L}^{2}(N)\right)_{N}$ and

$$
\gamma\left(u_{h}\right)=\sum_{i=1}^{n} e_{i i} \otimes u_{g_{i}}^{*} u_{h} u_{g_{i}} \quad \text { for all } h \in \Lambda_{0} .
$$

We now prove the following three statements. 1) If ${ }_{N} \mathcal{H}_{N}$ is of good form, then the same holds for all its $N$-N-subbimodules. 2) If ${ }_{N} \mathcal{H}_{N}$ and ${ }_{N} \mathcal{H}^{\prime}{ }_{N}$ are both of good form, then $\mathcal{H}^{\prime} \otimes_{N} \mathcal{H}$ is again of good form. 3) We can decompose $\mathrm{L}^{2}\left(M_{0}\right)$ as a direct sum of $N$-N-subbimodules of good form.

It is obvious that being of good form is preserved by direct sums. Furthermore, because ${ }_{N} \mathrm{~L}^{2}\left(M_{0}\right)_{N}$ is isomorphic with its contragredient, $\mathcal{F}_{0}$ is the smallest set of finite index $N$ - $N$-bimodules (up to isomorphism), containing the finite index $N$-N-subbimodules of $\mathrm{L}^{2}\left(M_{0}\right)$ and being stable under 
direct sums, tensor products and subbimodules. Hence, once statements 1, 2 and 3 are proven, our claim is proven as well.

Proof of 1). Let $\gamma, g_{1}, \ldots, g_{n} \in \Gamma$ and $\Lambda_{0}$ be given as above. If $h_{1}, \ldots, h_{n} \in \Lambda$ and if we replace $g_{i}$ by $g_{i} h_{i}$ and $\gamma$ by $a \mapsto W^{*} \gamma(a) W$, where $W \in N^{n}$ is given by $W:=\sum_{i=1}^{n} e_{i i} \otimes u_{h_{i}}$, formula (3) still holds. So, we may assume that whenever $g_{j} \in g_{i} \Lambda$, then $g_{j}=g_{i}$. Let $s_{1}, \ldots, s_{k}$ be the distinct elements of $\Gamma$ such that $\left\{g_{1}, \ldots, g_{n}\right\}=\left\{s_{1}, \ldots, s_{k}\right\}$. By construction, $s_{j} \notin s_{i} \Lambda$ whenever $i \neq j$. We have obvious diagonal projections $p_{i} \in \mathrm{M}_{n}(\mathbb{C})$, summing to 1 and satisfying

$$
\gamma\left(u_{h}\right)=\sum_{i=1}^{k} p_{i} \otimes u_{s_{i}}^{*} u_{h} u_{s_{i}} .
$$

Put $Z:=\sum_{i=1}^{k} p_{i} \otimes u_{s_{i}}$, which is a unitary in $M_{0}^{n}$. It follows that $\gamma\left(u_{h}\right)=Z^{*}\left(1 \otimes u_{h}\right) Z$ for all $h \in \Lambda_{0}$ and hence,

$$
\gamma(N)^{\prime} \cap N^{n} \subset Z^{*}\left(\left(1 \otimes \mathrm{L}_{\Omega}\left(\Lambda_{0}\right)\right)^{\prime} \cap M_{0}^{n}\right) Z \subset Z^{*}\left(\mathrm{M}_{n}(\mathbb{C}) \otimes 1\right) Z .
$$

Let now $q \in \gamma(N)^{\prime} \cap N^{n}$ be a projection. We have to prove that the $N$ - $N$-bimodule defined by $a \mapsto \gamma(a) q$ is again of good form. Because of (4), it suffices to prove that $q=r \otimes 1$, where $r \in \mathrm{M}_{n}(\mathbb{C})$ and $r p_{i}=p_{i} r$ for all $i=1, \ldots, k$. By $(5), q=Z^{*}(t \otimes 1) Z$ for some $t \in \mathrm{M}_{n}(\mathbb{C})$. Let $i \neq j$. Then, $\left(p_{i} \otimes 1\right) q\left(p_{j} \otimes 1\right)$ belongs to $\mathrm{M}_{n}(\mathbb{C}) \otimes N$ and to $\mathrm{M}_{n}(\mathbb{C}) \otimes \mathbb{C} u_{s_{i}^{-1} s_{j}}$. Hence, $\left(p_{i} \otimes 1\right) q\left(p_{j} \otimes 1\right)=0$. But then,

$$
q=\sum_{i=1}^{k}\left(p_{i} \otimes 1\right) q\left(p_{i} \otimes 1\right)=\sum_{i=1}^{k} p_{i} t p_{i} \otimes 1,
$$

concluding the proof of 1 ).

Proof of 2). Suppose that ${ }_{N} \mathcal{H}_{N}$ and ${ }_{N} \mathcal{H}^{\prime}{ }_{N}$ are of good form. Suppose that $\gamma: N \rightarrow N^{n}$ defines ${ }_{N} \mathcal{H}_{N}$ and satisfies (3) with respect to $g_{1}, \ldots, g_{n}, \Lambda_{0}<\Lambda \cap \bigcap_{i=1}^{n} g_{i} \Lambda g_{i}^{-1}$. Suppose that $\rho: N \rightarrow N^{m}$ defines ${ }_{N} \mathcal{H}^{\prime}{ }_{N}$ and satisfies (3) with respect to $h_{1}, \ldots, h_{m}$ and $\Lambda_{1}<\Lambda \cap \bigcap_{j=1}^{m} h_{j} \Lambda h_{j}^{-1}$. Then the composition $(\mathrm{id} \otimes \gamma) \rho$ satisfies (3) with respect to the group elements $h_{j} g_{i}$ and the subgroup $\Lambda_{1} \cap \bigcap_{j=1}^{m} h_{j} \Lambda_{0} h_{j}^{-1}$. It follows that $\mathcal{H}^{\prime} \otimes_{N} \mathcal{H}$ is of good form.

Proof of 3). For every $g \in \Gamma$, define $\mathcal{H}(g)$ as the closure of $N u_{g} N$ in $\mathrm{L}^{2}\left(M_{0}\right)$. Write

$$
\Lambda g \Lambda=\bigsqcup_{i=1}^{n} g_{i} \Lambda \text {. }
$$

The map $e_{i} \otimes a \rightarrow u_{g_{i}} a$ extends to a unitary $V: \mathbb{C}^{n} \otimes \mathrm{L}^{2}(N) \rightarrow \mathcal{H}(g)$ satisfying $V(\xi a)=V(\xi) a$ for all $\xi \in \mathbb{C}^{n} \otimes \mathrm{L}^{2}(N)$ and $a \in N$. Define $\gamma: N \rightarrow N^{n}$ such that $V(\gamma(a) \xi)=a V(\xi)$ for all $\xi \in \mathbb{C}^{n} \otimes \mathrm{L}^{2}(N)$ and $a \in N$. One checks that $\gamma$ satisfies (3) with respect to $g_{1}, \ldots, g_{n}$ and the subgroup $\Lambda_{0}=\Lambda \cap \bigcap_{i=1}^{n} g_{i} \Lambda g_{i}^{-1}$. Since the linear span of all $\mathcal{H}(g), g \in \Gamma$, is dense in $\mathrm{L}^{2}\left(M_{0}\right)$, the final statement 3 ) is proven and hence, also our claim.

Fix now an irreducible finite index $M_{0}-M_{0}$-bimodule $M_{0} \mathcal{K}_{M_{0}}$ and let $\mathcal{H} \subset \mathcal{K}$ be an irreducible $N$ $N$-subbimodule such that ${ }_{N} \mathcal{H}_{N}$ belongs to $\mathcal{F}_{0}$. By Lemma 2.2, for every $g, h \in \Gamma$, the closure of $N u_{g} \cdot \mathcal{H} \cdot u_{h} N$ inside $\mathcal{K}$ is isomorphic with an $N$ - $N$-subbimodule of $\mathcal{H}(g) \otimes_{N} \mathcal{H} \otimes_{N} \mathcal{H}(h)$. Hence, this closure belongs to $\mathcal{F}_{0}$ and the irreducibility of $\mathcal{K}$ implies that $\mathcal{K}$ is a direct sum of $N$-N-subbimodules that all belong to $\mathcal{F}_{0}$.

Write $M_{0} \mathcal{K}_{M_{0}} \cong \psi\left(M_{0}\right) p\left(\mathbb{C}^{m} \otimes \mathrm{L}^{2}\left(M_{0}\right)\right)_{M_{0}}$ for some finite index irreducible inclusion $\psi: M_{0} \rightarrow$ $p M_{0}^{m} p$. Define $A:=p M_{0}^{m} p \cap \psi(N)^{\prime}$. Since $\mathcal{K}$ is of finite index and $N \subset M_{0}$ is irreducible, it follows 
that $A$ is finite dimensional. Let $q \in A$ be a minimal projection. Because of the previous paragraph, we find a finite index inclusion $\gamma: N \rightarrow N^{n}$ satisfying (3) with respect to $g_{1}, \ldots, g_{n}$ and $\Lambda_{0}$ and a bimodular isometry

$$
\theta: \gamma(N)\left(\mathbb{C}^{n} \otimes \mathrm{L}^{2}(N)\right)_{N} \rightarrow \psi(N) q q\left(\mathbb{C}^{m} \otimes \mathrm{L}^{2}\left(M_{0}\right)\right)_{N}
$$

Define $\xi \in q\left(\mathbb{C}^{m}\left(\mathbb{C}^{n}\right)^{*} \otimes \mathrm{L}^{2}\left(M_{0}\right)\right)$ by the formula

$$
\xi:=\sum_{i=1}^{n} \theta\left(e_{i} \otimes 1\right)\left(e_{i}^{*} \otimes 1\right) .
$$

It follows that $\xi$ is non-zero and satisfies $\psi(a) \xi=\xi \gamma(a)$ for all $a \in N$. As an element of $q\left(\mathrm{M}_{m}(\mathbb{C}) \otimes\right.$ $\left.\mathrm{L}^{1}\left(M_{0}\right)\right) q$, the operator $\xi \xi^{*}$ commutes with $\psi(N)$. Hence, $\xi \xi^{*}$ is a multiple of $q$ and we may assume that $\xi \in q\left(\mathbb{C}^{m}\left(\mathbb{C}^{n}\right)^{*} \otimes M_{0}\right)$ with $\xi \xi^{*}=q$. Define $V=\xi\left(\sum_{i} e_{i i} \otimes u_{g_{i}}^{*}\right)$. It follows that $V \in q\left(\mathbb{C}^{m}\left(\mathbb{C}^{n}\right)^{*} \otimes M_{0}\right), V V^{*}=q$ and $\psi\left(u_{h}\right) V=V\left(1 \otimes u_{h}\right)$ for all $h \in \Lambda_{0}$. Since $\mathrm{L}_{\Omega}\left(\Lambda_{0}\right)$ has trivial relative commutant in $M_{0}$, it follows that $V^{*} V=p_{1} \otimes 1$ for some projection $p_{1} \in \mathrm{M}_{n}(\mathbb{C})$. Hence, we can find $W \in q\left(\mathbb{C}^{m}\left(\mathbb{C}^{k}\right)^{*} \otimes M_{0}\right)$ satisfying $W W^{*}=q, W^{*} W=1$ and $\psi\left(u_{h}\right) W=W\left(1 \otimes u_{h}\right)$ for all $h$ in a finite index subgroup of $\Lambda$.

Repeating the same procedure for a set of minimal projections in $A$ summing to 1 and taking the intersection of all finite index subgroups of $\Lambda$ involved, we find a unitary $X \in p\left(\mathbb{C}^{m}\left(\mathbb{C}^{r}\right)^{*} \otimes M_{0}\right)$ and a finite index subgroup $\Lambda_{0}<\Lambda$ satisfying $X^{*} \psi\left(u_{h}\right) X=1 \otimes u_{h}$ for all $h \in \Lambda_{0}$. So, we may actually assume that $\psi\left(u_{h}\right)=1 \otimes u_{h}$ for all $h \in \Lambda_{0}$.

If now $g \in \Gamma$, we get that $\psi\left(u_{g}\right)\left(1 \otimes u_{g}^{*}\right)$ commutes with $1 \otimes u_{h}$ for all $h \in \Lambda_{0} \cap g \Lambda_{0} g^{-1}$. Hence, $\psi\left(u_{g}\right)=\pi(g) \otimes u_{g}$ for all $g \in \Gamma$, where $\pi: \Gamma \rightarrow \mathcal{U}\left(\mathbb{C}^{r}\right)$ is a finite dimensional unitary representation. By assumption, $\pi(g)=1$ for all $g \in \Gamma$. So, $\psi(a)=1 \otimes a$ for all $a \in M_{0}$. By irreducibility of $\mathcal{K}$, we get $r=1$ and ${ }_{M_{0}} \mathcal{K}_{M_{0}} \cong{ }_{M_{0}} \mathrm{~L}^{2}\left(M_{0}\right)_{M_{0}}$.

In fact, we only use the following concrete example satisfying the conditions of Lemma 3.3 and hence providing an inclusion $N \subset M_{0}$ satisfying assumption 2, with $N$ being isomorphic with the hyperfinite $\mathrm{II}_{1}$ factor. Moreover $N \subset M_{0}$ satisfies assumption 3 .

Example 3.4. Consider the group $\Gamma=\left(\mathbb{Q}^{3} \oplus \mathbb{Q}^{3}\right) \rtimes \mathrm{SL}(3, \mathbb{Q})$, defined by the action $A \cdot(x, y)=$ $\left(A x,\left(A^{t}\right)^{-1} y\right)$ of $\mathrm{SL}(3, \mathbb{Q})$ on $\mathbb{Q}^{3} \oplus \mathbb{Q}^{3}$. Choose an irrational number $\alpha \in \mathbb{R}$ and define $\Omega \in \mathrm{Z}^{2}\left(\Gamma, S^{1}\right)$ such that

$$
\begin{aligned}
\Omega\left((x, y),\left(x^{\prime}, y^{\prime}\right)\right) & =\exp \left(i \alpha\left(\left\langle x, y^{\prime}\right\rangle-\left\langle y, x^{\prime}\right\rangle\right)\right) \quad \text { for all }(x, y),\left(x^{\prime}, y^{\prime}\right) \in \mathbb{Q}^{3} \oplus \mathbb{Q}^{3}, \\
\Omega(g, A) & =\Omega(A, g)=\Omega(A, B)=1 \quad \text { for all } g \in \Gamma, A, B \in \operatorname{SL}(3, \mathbb{Q})
\end{aligned}
$$

Set $\Lambda=\mathbb{Z}^{3} \oplus \mathbb{Z}^{3}$. We define $N:=\mathrm{L}_{\Omega}(\Lambda)$ and $M_{0}:=\mathrm{L}_{\Omega}(\Gamma)$. We prove that $N \subset M_{0}$ satisfies assumptions 2 and 3 .

Assumption 3 follows by taking $N_{0}:=\mathrm{L}_{\Omega}\left(\left(\mathbb{Z}^{3} \oplus \mathbb{Z}^{3}\right) \rtimes \mathrm{SL}(3, \mathbb{Z})\right)$, which has property $(\mathrm{T})$ because $\left(\mathbb{Z}^{3} \oplus \mathbb{Z}^{3}\right) \rtimes \mathrm{SL}(3, \mathbb{Z})$ is a property $(\mathrm{T})$ group.

Since $\operatorname{SL}(3, \mathbb{Q})$ has no non-trivial finite-dimensional unitary representations and since the smallest normal subgroup of $\Gamma$ containing $\operatorname{SL}(3, \mathbb{Q})$ is the whole of $\Gamma$, it follows that $\Gamma$ has no non-trivial finite-dimensional unitary representations. Because of Lemma 3.3, it remains to prove that for every finite index subgroup $\Lambda_{0}<\Lambda$, we have $\mathrm{L}_{\Omega}(\Gamma) \cap \mathrm{L}_{\Omega}\left(\Lambda_{0}\right)^{\prime}=\mathbb{C} 1$. Write $\Lambda_{1}=\mathbb{Q}^{3} \oplus \mathbb{Q}^{3}$. Take $a \in \mathrm{L}_{\Omega}(\Gamma) \cap \mathrm{L}_{\Omega}\left(\Lambda_{0}\right)^{\prime}$ and write, with $\mathrm{L}^{2}$-convergence, $a=\sum_{g \in \Gamma} a_{g} u_{g}$. Since

$$
\left\{h g h^{-1} \mid h \in \Lambda_{0}\right\}
$$


is infinite for all $g \in \Gamma-\Lambda_{1}$, we immediately get $a_{g}=0$ for all $g \in \Gamma-\Lambda_{1}$. On the other hand, define

$$
\pi: \Lambda_{1} \rightarrow \widehat{\Lambda_{1}}: h \mapsto \pi_{h} \quad \text { where } \pi_{h}(g)=\Omega(h, g)^{-2} .
$$

It follows that $a_{g} \pi_{g}(h)=a_{g}$ for all $g \in \Lambda_{1}$ and all $h \in \Lambda_{0}$. If $g \in \Lambda_{1}-\{0\}$, the character $\pi_{g}$ is not identically 1 on $\Lambda_{0}$ and hence, $a_{g}=0$. It follows that $a \in \mathbb{C} 1$.

We deduce Theorem 1.1 from the following general statement.

Theorem 3.5. Under the assumptions at the beginning of the section and writing $M=M_{0} *_{N} M_{1}$, the action $G \curvearrowright M$ is minimal and the natural tensor functor defined in 2.4

$$
\operatorname{Rep}(G) \rightarrow \operatorname{Bimod}\left(M^{G}\right): \pi \mapsto M^{G} \operatorname{Mor}\left(H_{\pi}, \mathrm{L}^{2}(M)\right)_{M^{G}}
$$

is an equivalence of categories.

The rest of this section is devoted to a proof of Theorem 3.5 and deducing 1.1 as a corollary. Denote $P=M^{G}$ and make throughout the assumptions made at the beginning of the section.

Choose a complete set $\operatorname{Irr}(G)$ of representatives for the set of irreducible unitary representations of $G$ modulo unitary conjugacy. Since $G \stackrel{\sigma}{\curvearrowright} M_{1}$ is minimal, choose, for every $\pi \in \operatorname{Irr}(G)$, a unitary $V_{\pi} \in \mathrm{B}\left(H_{\pi}\right) \otimes M_{1}$ satisfying (id $\left.\otimes \sigma_{g}\right)\left(V_{\pi}\right)=V_{\pi}(\pi(g) \otimes 1)$ for all $g \in G$. Define the finite index inclusions

$$
\psi_{\pi}: P \rightarrow \mathrm{B}\left(H_{\pi}\right) \otimes P: \psi_{\pi}(a)=V_{\pi}(1 \otimes a) V_{\pi}^{*} \text { for all } a \in P
$$

and note that $\psi_{\pi}(N) \subset \mathrm{B}\left(H_{\pi}\right) \otimes N$. As before, we denote by $H\left(\psi_{\pi}\right)$ the $N$ - $N$-bimodule given by ${ }_{N}\left(H_{\pi}^{*} \otimes \mathrm{L}^{2}(N)\right)_{\psi_{\pi}(N)}$.

Remark 3.6. In Section 2.7, we explained that the $N$ - $N$-bimodule ${ }_{N} \mathrm{~L}^{2}\left(M_{1}\right)_{N}$ can be decomposed into a direct sum of $N$-N-bimodules $L(\pi), \pi \in \operatorname{Irr}(G)$, where $L(\pi)$ is isomorphic with $\operatorname{dim}(\pi)$ copies of $H\left(\psi_{\pi}\right)$. Furthermore $L(\pi)$ is the closure of $L^{0}(\pi) \subset M_{1}$, in such a way that the linear span of all $L^{0}(\pi)$ is an ultraweakly dense $*$-subalgebra of $M_{1}$.

Since $N \subset M_{0}$ is irreducible and quasi-regular, we can decompose $\mathrm{L}^{2}\left(M_{0}\right) \ominus \mathrm{L}^{2}(N)$ as the orthogonal direct sum of irreducible, non-trivial, finite index $N$ - $N$-subbimodules $R_{i}, i \in I$. Put $R_{i}^{0}:=R_{i} \cap M_{0}$. By Lemma 2.2, $R_{i}^{0}$ is dense in $R_{i}$. By construction, $R_{i}^{0} \subset M_{0} \ominus N$. Assume $0 \notin I$ and put $R_{0}^{0}:=N$. By Lemma 2.3, $\operatorname{span}\left\{R_{i}^{0} \mid i \in I \cup\{0\}\right\}$ equals $\mathrm{QN}_{M_{0}}(N)$ and is, in particular, an ultraweakly dense *-subalgebra of $M_{0}$.

Whenever $n \in \mathbb{N} \cup\{0\}, i_{0}, i_{n} \in I \cup\{0\}, i_{1}, \ldots, i_{n-1} \in I$ and $\pi_{1}, \ldots, \pi_{n} \in \operatorname{Irr}(G) \backslash\{\epsilon\}$, denote by $R\left(i_{0}, \pi_{1}, \ldots, \pi_{n}, i_{n}\right)$ the closure of

$$
R\left(i_{0}, \pi_{1}, \ldots, \pi_{n}, i_{n}\right)^{0}:=R_{i_{0}}^{0} L^{0}\left(\pi_{1}\right) R_{i_{1}}^{0} \cdots R_{i_{n-1}}^{0} L^{0}\left(\pi_{n}\right) R_{i_{n}}^{0}
$$

inside $\mathrm{L}^{2}(M)$. The definition of the amalgamated free product implies that $\mathrm{L}^{2}(M)$ is the orthogonal direct sum of the subspaces $R\left(i_{0}, \pi_{1}, \ldots, \pi_{n}, i_{n}\right)$. Furthermore, the freeness with amalgamation implies that the map

$$
a_{0} \otimes b_{1} \otimes a_{1} \otimes \cdots \otimes b_{n} \otimes a_{n} \mapsto a_{0} b_{1} a_{1} \cdots b_{n} a_{n}
$$

extends to a unitary from $R_{i_{0}} \otimes_{N} L\left(\pi_{1}\right) \otimes_{N} \cdots \otimes_{N} L\left(\pi_{n}\right) \otimes_{N} R_{i_{n}}$ onto $R\left(i_{0}, \pi_{1}, \ldots, \pi_{n}, i_{n}\right)$. In particular, $R\left(i_{0}, \pi_{1}, \ldots, \pi_{n}, i_{n}\right)$ is isomorphic with $\operatorname{dim}\left(\pi_{1}\right) \cdots \operatorname{dim}\left(\pi_{n}\right)$ copies of

$$
R_{i_{0}} \otimes_{N} H\left(\psi_{\pi_{1}}\right) \otimes_{N} \cdots \otimes_{N} H\left(\psi_{\pi_{n}}\right) \otimes_{N} R_{i_{n}} .
$$


Finally, the linear span of all $R\left(i_{0}, \pi_{1}, \ldots, \pi_{n}, i_{n}\right)^{0}$ is an ultraweakly dense $*$-subalgebra of $M$.

Denote by $\mathcal{F}_{0}$ the fusion subalgebra of $\operatorname{FAlg}(N)$ generated by the finite index $N$ - $N$-subbimodules $R_{i}, i \in I$. Assumption 4 implies in particular that the fusion subalgebra of $\mathrm{FAlg}(N)$ generated by $H\left(\psi_{\pi}\right), \pi \in \operatorname{Irr}(G)$, is free w.r.t. $\mathcal{F}_{0}$. Therefore the $N$-N-bimodules appearing in (6) are irreducible and we have found a decomposition of ${ }_{N} \mathrm{~L}^{2}(M)_{N}$ as a direct sum of irreducible finite index $N-N$ bimodules. The trivial $N-N$-bimodule appears with multiplicity one in $\mathrm{L}^{2}(M)$. This means that $N^{\prime} \cap M=\mathbb{C} 1$. In particular, the action $G \curvearrowright M$ is minimal.

The action of $G$ on $\mathrm{L}^{2}(M)$ leaves every $R\left(i_{0}, \pi_{1}, \ldots, \pi_{n}, i_{n}\right)$ globally invariant and we denote by $R\left(i_{0}, \pi_{1}, \ldots, \pi_{n}, i_{n}\right)^{G}$ the subspace of $G$-fixed vectors. It follows that $\mathrm{L}^{2}(P)$ is the orthogonal direct sum of the $N$ - $N$-subbimodules $R\left(i_{0}, \pi_{1}, \ldots, \pi_{n}, i_{n}\right)^{G}$, which are, as $N$ - $N$-bimodules isomorphic with a multiple of the $N$-N-bimodule given by (6). This multiple is in its turn given by the dimension of the space of $\left(\pi_{1} \otimes \cdots \otimes \pi_{n}\right)(G)$-invariant vectors in $H_{\pi_{1}} \otimes \cdots \otimes H_{\pi_{n}}$.

Lemma 3.7. If $M_{0} \mathcal{H}_{P}$ is an irreducible non-zero $M_{0}$-P-bimodule with $\operatorname{dim}\left(\mathcal{H}_{P}\right)<\infty$, there exists $\eta \in \operatorname{Irr}(G)$ and a non-zero $M_{0}-\psi_{\eta}\left(M_{0}\right)$-subbimodule $\mathcal{K} \subset H_{\eta}^{*} \otimes \mathcal{H}$ with the following properties.

- $\operatorname{dim}\left(\mathcal{K}_{\psi_{\eta}\left(M_{0}\right)}\right)<\infty$.

- If $\rho \in \operatorname{Irr}(G)$ and $\mathcal{L} \subset H_{\rho}^{*} \otimes \mathcal{H}$ is a non-zero $M_{0}-\psi_{\rho}\left(M_{0}\right)$-subbimodule with $\operatorname{dim}\left(M_{0} \mathcal{L}\right)<\infty$, then $\rho=\eta$ and $\mathcal{L} \subset \mathcal{K}$.

Proof. Take $\psi: M_{0} \rightarrow p P^{n} p$ such that ${ }_{M_{0}} \mathcal{H}_{P} \cong{ }_{\psi\left(M_{0}\right)} p\left(\mathbb{C}^{n} \otimes \mathrm{L}^{2}(P)\right)_{P}$. By assumption, the inclusion $\psi\left(M_{0}\right) \subset p P^{n} p$ is irreducible. From assumption 3 , we get the property $(\mathrm{T}) \mathrm{II}_{1}$ factor $N_{0}$ and hence, $\psi\left(N_{0}\right)$ has property $(\mathrm{T})$ and is a subalgebra of $p P^{n} p \subset p M^{n} p$. Recall that $M=M_{0} *_{N} M_{1}$ and that $M_{1}$ is hyperfinite. Since there is no non-zero homomorphism from a property $(\mathrm{T}) \mathrm{II}_{1}$ factor to the hyperfinite $\mathrm{II}_{1}$ factor, 2.10 .2 provides $u \in p\left(\mathbb{C}^{n}\left(\mathbb{C}^{k}\right)^{*} \otimes M\right)$ with $u u^{*}=p, q:=u^{*} u \in M_{0}^{k}$ and $u^{*} \psi\left(N_{0}\right) u \subset q M_{0}^{k} q$. Since $N_{0} \subset M_{0}$ is quasi-regular, 2.10.1 implies that $u^{*} \psi\left(M_{0}\right) u \subset q M_{0}^{k} q$.

Define $\gamma: M_{0} \rightarrow q M_{0}^{k} q: \gamma(a)=u^{*} \psi(a) u$. We now use the bimodule maps $E_{\pi}$ given by (2). Take $\eta \in \operatorname{Irr}(G)$ such that $\left(\right.$ id $\left.\otimes E_{\eta}\right)(u) \neq 0$. So, we get a non-zero $v \in p\left(\mathbb{C}^{n}\left(\mathbb{C}^{k} \otimes H_{\eta}\right)^{*} \otimes P\right)$ satisfying

$$
\psi(a) v=v\left(\operatorname{id} \otimes \psi_{\eta}\right) \gamma(a)
$$

for all $a \in M_{0}$. Replacing $v$ by its polar part, we may assume that $v$ is a partial isometry. The irreducibility of $\psi\left(M_{0}\right) \subset p P^{n} p$ ensures that $v v^{*}=p$.

Define the $\psi\left(M_{0}\right)-\psi_{\eta}\left(M_{0}\right)$-subbimodule $\mathcal{K}$ of $p\left(\mathbb{C}^{n} H_{\eta}^{*} \otimes \mathrm{L}^{2}(P)\right)$ as the closure of $v\left(\mathbb{C}^{k} \otimes \psi_{\eta}\left(M_{0}\right)\right)$. By construction, $\operatorname{dim}\left(\mathcal{K}_{\psi_{\eta}\left(M_{0}\right)}\right)<\infty$.

Let $\rho \in \operatorname{Irr}(G)$ and let $\mathcal{L} \subset p\left(\mathbb{C}^{n} H_{\rho}^{*} \otimes \mathrm{L}^{2}(P)\right)$ be a non-zero $\psi\left(M_{0}\right)-\psi_{\rho}\left(M_{0}\right)$-subbimodule with $\operatorname{dim}\left(\psi\left(M_{0}\right) \mathcal{L}\right)<\infty$. We have to prove that $\rho=\eta$ and $\mathcal{L} \subset \mathcal{K}$. Since $\operatorname{dim}\left(\psi\left(M_{0}\right) \mathcal{L}\right)<\infty$, we can take a non-zero vector

$$
\xi \in(1 \otimes p)\left(\left(\mathbb{C}^{l} \otimes \mathbb{C}^{n}\right) H_{\rho}^{*} \otimes \mathrm{L}^{2}(P)\right)
$$

and a, possibly non-unital, $*$-homomorphism $\theta: M_{0} \rightarrow M_{0}^{l}$ satisfying $\xi \psi_{\rho}(a)=(\operatorname{id} \otimes \psi) \theta(a) \xi$ for all $a \in M_{0}$ and such that $\mathcal{L}$ is the closed linear span of $\left(\left(\mathbb{C}^{l}\right)^{*} \otimes \psi\left(M_{0}\right)\right) \xi$.

Put $\zeta=\left(1 \otimes 1 \otimes V_{\eta}^{*}\right)\left(1 \otimes v^{*}\right) \xi V_{\rho}$. Since $v v^{*}=p$, we know that $\zeta$ is non-zero. Then,

$$
\zeta \in\left(\mathbb{C}^{l} \otimes \mathbb{C}^{k} \otimes H_{\eta}\right) H_{\rho}^{*} \otimes \mathrm{L}^{2}(M)
$$


and $\zeta$ satisfies $\zeta(1 \otimes a)=((\mathrm{id} \otimes \gamma) \theta(a))_{124} \zeta$ for all $a \in M_{0}$. By Theorem 2.10.1, it follows that $\zeta \in\left(\mathbb{C}^{l} \otimes \mathbb{C}^{k} \otimes H_{\eta}\right) H_{\rho}^{*} \otimes \mathrm{L}^{2}\left(M_{0}\right)$. In particular, $\zeta$ is $G$-invariant. Since $\zeta=\left(1 \otimes 1 \otimes V_{\eta}^{*}\right)\left(1 \otimes v^{*}\right) \xi V_{\rho}$ and $\xi$ is a non-zero $G$-invariant vector, it follows that $\eta=\rho$ and $\zeta=\left(\zeta_{0}\right)_{124}$ for some $\zeta_{0} \in \mathbb{C}^{l} \otimes \mathbb{C}^{k} \otimes \mathrm{L}^{2}\left(M_{0}\right)$. It finally follows that

$$
\xi=(1 \otimes v)\left(\mathrm{id} \otimes \mathrm{id} \otimes \psi_{\eta}\right)\left(\zeta_{0}\right)
$$

which belongs to $\mathbb{C}^{l} \otimes \mathcal{K}$, ending the proof of the lemma.

Lemma 3.8. Let ${ }_{P} \mathcal{H}_{P}$ be a finite index $P$-P-bimodule. For every non-zero irreducible $M_{0}-P$ subbimodule $\mathcal{H}_{0} \subset \mathcal{H}$, there exists $\eta \in \operatorname{Irr}(G)$ and a non-zero $M_{0}-\psi_{\eta}\left(M_{0}\right)$-subbimodule $\mathcal{K} \subset H_{\eta}^{*} \otimes \mathcal{H}_{0}$ such that ${ }_{M_{0}} \mathcal{K}_{\psi_{\eta}\left(M_{0}\right)}$ has finite index.

Proof. For every $\pi \in \operatorname{Irr}(G)$, define the finite index $P$ - $P$-bimodule $\mathcal{H}^{\pi}$ given by $\psi_{\pi}(P)\left(H_{\pi} \otimes \mathcal{H}\right)_{P}$. Since $M_{0} \subset P$ is irreducible, we find for every $\pi \in \operatorname{Irr}(G)$, a finite number $n_{\pi}$ and an orthogonal decomposition $\mathcal{H}^{\pi}=\bigoplus_{i=1}^{n_{\pi}} \mathcal{H}^{\pi, i}$ of $\mathcal{H}^{\pi}$ into irreducible $M_{0}-P$-bimodules. For every $\pi$, $i$, take $\eta_{\pi, i} \in \operatorname{Irr}(G)$ and a $\psi_{\pi}\left(M_{0}\right)-\psi_{\eta_{\pi, i}}\left(M_{0}\right)$-subbimodule $\mathcal{K}^{\pi, i} \subset H_{\eta_{\pi, i}}^{*} \otimes \mathcal{H}^{\pi, i}$ satisfying the conclusions of Lemma 3.7. Note that $\mathcal{K}^{\pi, i} \subset H_{\pi} H_{\eta_{\pi, i}}^{*} \otimes \mathcal{H}$.

Define the subset $J \subset \operatorname{Irr}(G) \times \operatorname{Irr}(G)$ consisting of $(\pi, \eta)$ for which there exists $1 \leq i \leq n_{\pi}$ with $\eta_{\pi, i}=\eta$. Moreover, define $\mathcal{K}^{\pi, \eta}=\operatorname{span}\left\{\mathcal{K}^{\pi, i} \mid \eta_{\pi, i}=\eta\right\}$. By construction, $\mathcal{K}^{\pi, \eta}$ is a non-zero $\psi_{\pi}\left(M_{0}\right)-\psi_{\eta}\left(M_{0}\right)$-subbimodule of $H_{\pi} H_{\eta}^{*} \otimes \mathcal{H}$, of finite right $\psi_{\eta}\left(M_{0}\right)$-dimension. Moreover, whenever $\pi, \eta \in \operatorname{Irr}(G)$ and $\mathcal{K} \subset H_{\pi} H_{\eta}^{*} \otimes \mathcal{H}$ is a $\psi_{\pi}\left(M_{0}\right)-\psi_{\eta}\left(M_{0}\right)$-subbimodule of finite left $\psi_{\pi}\left(M_{0}\right)$-dimension, it follows that $(\pi, \eta) \in J$ and $\mathcal{K} \subset \mathcal{K}^{\pi, \eta}$.

By symmetry, we also find a subset $J^{\prime} \subset \operatorname{Irr}(G) \times \operatorname{Irr}(G)$ and for all $(\pi, \eta) \in J^{\prime}$ a $\psi_{\pi}\left(M_{0}\right)-\psi_{\eta}\left(M_{0}\right)$ subbimodule $\mathcal{L}^{\pi, \eta}$ of $H_{\pi} H_{\eta}^{*} \otimes \mathcal{H}$ which is of finite left $\psi_{\pi}\left(M_{0}\right)$-dimension and which has the following property: if $\pi, \eta \in \operatorname{Irr}(G)$ and $\mathcal{L} \subset H_{\pi} H_{\eta}^{*} \otimes \mathcal{H}$ is a non-zero $\psi_{\pi}\left(M_{0}\right)-\psi_{\eta}\left(M_{0}\right)$-subbimodule of finite right $\psi_{\eta}\left(M_{0}\right)$-dimension, we have $(\pi, \eta) \in J^{\prime}$ and $\mathcal{L} \subset \mathcal{L}^{\pi, \eta}$.

But then, $J=J^{\prime}$ and $\mathcal{K}^{\pi, \eta}=\mathcal{L}^{\pi, \eta}$ for all $(\pi, \eta) \in J=J^{\prime}$. Hence, all $\mathcal{K}^{\pi, \eta}$ are finite index $\psi_{\pi}\left(M_{0}\right)$ $\psi_{\eta}\left(M_{0}\right)$-bimodules. To conclude the proof of the lemma, it suffices to observe that $\bigoplus_{i=1}^{n_{\epsilon}} \mathcal{H}^{\epsilon, i}$ is a decomposition of $\mathcal{H}$ into irreducible $M_{0}-P$-subbimodules and that $\mathcal{K}^{\epsilon, i} \subset H_{\eta_{\epsilon, i}}^{*} \otimes \mathcal{H}^{\epsilon, i}$ is the required finite index $M_{0}-\psi_{\eta_{\epsilon, i}}\left(M_{0}\right)$-subbimodule.

Lemma 3.9. Let ${ }_{P} \mathcal{H}_{P}$ be a finite index $P$-P-bimodule and $\mathcal{K} \subset \mathcal{H}$ a non-zero irreducible $M_{0}-M_{0}$ subbimodule such that ${ }_{M_{0}} \mathcal{K}_{M_{0}}$ has finite index. Then, $\mathcal{K}$ is the trivial $M_{0}-M_{0}$-bimodule: ${ }_{M_{0}} \mathcal{K}_{M_{0}} \cong$ $M_{0} \mathrm{~L}^{2}\left(M_{0}\right)_{M_{0}}$.

Proof. We may assume that ${ }_{P} \mathcal{H}_{P}$ is irreducible.

Step 1. $\mathcal{K}$ contains a non-zero $N$-N-subbimodule $\mathcal{L}$ with $\operatorname{dim}\left(\mathcal{L}_{N}\right)<\infty$.

To prove step 1, take a finite index inclusion $\psi: P \rightarrow p P^{n} p$ such that ${ }_{P} \mathcal{H}_{P} \cong{ }_{\psi(P)} p\left(\mathbb{C}^{n} \otimes \mathrm{L}^{2}(P)\right)_{P}$. Let $\mathcal{K} \subset p\left(\mathbb{C}^{n} \otimes \mathrm{L}^{2}(P)\right)$ be an irreducible non-zero $\psi\left(M_{0}\right)-M_{0}$-subbimodule such that $\psi\left(M_{0}\right) \mathcal{K}_{M_{0}}$ has finite index. Take a finite index, irreducible inclusion $\rho: M_{0} \rightarrow q M_{0}^{k} q$ and a unitary

$$
\theta: q\left(\mathbb{C}^{k} \otimes \mathrm{L}^{2}\left(M_{0}\right)\right) \rightarrow \mathcal{K} \quad \text { s.t. } \quad \theta(\rho(a) \mu b)=\psi(a) \theta(\mu) b \quad \text { for all } \mu \in q\left(\mathbb{C}^{k} \otimes \mathrm{L}^{2}\left(M_{0}\right)\right), a, b \in M_{0} .
$$

Define the non-zero vector $\xi \in p\left(\mathbb{C}^{n}\left(\mathbb{C}^{k}\right)^{*} \otimes \mathrm{L}^{2}(P)\right) q$ by

$$
\xi=\sum_{i=1}^{k} \theta\left(q\left(e_{i} \otimes 1\right)\right)\left(e_{i}^{*} \otimes 1\right) .
$$


It follows that $\psi(a) \xi=\xi \rho(a)$ for all $a \in M_{0}$. As an element of $p\left(\mathrm{M}_{n}(\mathbb{C}) \otimes \mathrm{L}^{1}(P)\right) p$, the operator $\xi \xi^{*}$ commutes with $\psi\left(M_{0}\right)$. Since $M_{0} \subset P$ is irreducible and $\psi(P) \subset p P^{n} p$ has finite index, the relative commutant $\psi\left(M_{0}\right)^{\prime} \cap p P^{n} p$ is finite dimensional. Hence, $\xi \xi^{*}$ is bounded and it follows that $\xi \in p\left(\mathbb{C}^{n}\left(\mathbb{C}^{k}\right)^{*} \otimes P\right) q$. The irreducibility of $\rho\left(M_{0}\right) \subset q M_{0}^{k} q$ implies that $E_{M_{0}}\left(\xi^{*} \xi\right)$ is a non-zero multiple of $q$. Denote by $v \in p\left(\mathbb{C}^{n}\left(\mathbb{C}^{k}\right)^{*} \otimes P\right) q$ the polar part of $\xi$. Note that $\psi(a) v=v \rho(a)$ for all $a \in M_{0}$.

We claim that $\rho(N) \prec_{M_{0}} N$. Suppose not. Then, Theorem 2.10.1 implies that the quasi-normalizer of $\rho(N)$ inside $q M^{k} q$ is contained in $q M_{0}^{k} q$. Since $N \subset P$ is quasi-regular, it follows that $v^{*} \psi(P) v \subset$ $q M_{0}^{k} q$. Denote by $A$ the von Neumann subalgebra of $p P^{n} p$ generated by $\psi(P)$ and $v v^{*}$. Write $q_{1}=v^{*} v$ and note that $q_{1} \in q M_{0}^{k} q$. Since $\psi(P) \subset A \subset p P^{n} p$, it follows that $A \subset p P^{n} p$ has finite index. But then, $v^{*} A v$ is a finite index subalgebra of $q_{1} P^{k} q_{1}$. Since $v^{*} A v \subset q_{1} M_{0}^{k} q_{1}$ and $M_{0} \subset P$ has infinite index, we have reached a contradiction. This proves the claim.

The claim and the remark following Definition 2.9 yield $b_{1}, \ldots, b_{m} \in q\left(\mathbb{C}^{k} \otimes M_{0}\right)$ such that writing $V=\operatorname{span}\left\{b_{i} N \mid i=1, \ldots, m\right\}$, we have $V \neq\{0\}$ and $\rho(N) V=V$. Define $\mathcal{L} \subset \mathcal{K}$ as the closure of $\operatorname{span}\left\{\xi b_{i} N \mid i=1, \ldots, m\right\}$. By construction $\mathcal{L}$ is a $\psi(N)$ - $N$-subbimodule of $\mathcal{K}$ with $\operatorname{dim}\left(\mathcal{L}_{N}\right)<\infty$. Since $E_{M_{0}}\left(\xi^{*} \xi\right)$ is a multiple of $q$, it also follows that $\mathcal{L}$ is non-zero. So, Step 1 is proven.

Step 2. $\mathcal{K}$ is a direct sum of non-zero $N$ - $N$-subbimodules $\mathcal{L}$ such that ${ }_{N} \mathcal{L}_{N}$ has finite index.

By Step 1, take a non-zero $N$ - $N$-subbimodule $\mathcal{L}^{0}$ of $\mathcal{K}$ with $\operatorname{dim}\left(\mathcal{L}_{N}^{0}\right)<\infty$. For all $a, b \in \mathrm{QN}_{M_{0}}(N)$, the closure of $N a \cdot \mathcal{L}^{0} \cdot b N$ is still an $N$-N-subbimodule of $\mathcal{K}$ of finite right $N$-dimension. By irreducibility of ${ }_{M_{0}} \mathcal{K}_{M_{0}}$ and quasi-regularity of $N \subset M_{0}$, the linear span of all $N a \cdot \mathcal{L}^{0} \cdot b N$ is dense in $\mathcal{K}$. So, we have written $\mathcal{K}$ as a direct sum of $N$ - $N$-subbimodules of finite right $N$-dimension. By symmetry, we can also write $\mathcal{K}$ as a direct sum of $N$-N-subbimodules of finite left $N$-dimension. Taking all non-zero intersections of $N-N$-subbimodules of both kinds, we end the proof of Step 2.

Step 3. Define as above the fusion subalgebra $\mathcal{F}_{0}$ of $\operatorname{FAlg}(N)$ generated by the finite index $N-N$ subbimodules of $\mathrm{L}^{2}\left(M_{0}\right)$. We now prove that every irreducible $N$-N-subbimodule of $\mathcal{K}$ belongs to $\mathcal{F}_{0}$.

Choose an infinite set $K_{i}, i \in I$, of irreducible, non-trivial, inequivalent $N$ - $N$-bimodules that appear as $N$-N-subbimodules of $\mathrm{L}^{2}\left(M_{0}\right)$. Since $M_{0}$ is not hyperfinite, the inclusion $N \subset M_{0}$ has infinite index and hence, Lemma 2.1 implies that such an infinite set $I$ can be chosen. Denote $K_{i}^{0}:=K_{i} \cap M_{0}$. By Lemma $2.2, K_{i}^{0}$ is dense in $K_{i}$ and $K_{i}^{0}$ is finitely generated, both as a left and as a right $N$-module.

Assume by contradiction that $\mathcal{L} \subset \mathcal{K}$ is an irreducible $N$-N-subbimodule such that ${ }_{N} \mathcal{L}_{N}$ has finite index and $\mathcal{L} \notin \mathcal{F}_{0}$. Define $\mathcal{F}$ as in assumption 4 and note that by construction $\mathcal{L} \in \mathcal{F}$. Take some $\pi \in \operatorname{Irr}(G), \pi \neq \epsilon$. Take $\eta \in \operatorname{Irr}(G)$ unitarily equivalent with the contragredient representation of $\pi$. Let $\xi_{0} \in H_{\eta} \otimes H_{\pi}$ be a non-zero $(\eta \otimes \pi)$-invariant vector. Define for every $i \in I$, the subspace $\mathcal{T}_{i} \subset P$ given by

$$
\mathcal{T}_{i}:=\operatorname{span}\left(\left(H_{\eta} \otimes H_{\pi}\right)^{*} \otimes N\right)\left(V_{\eta}\right)_{13}\left(1 \otimes 1 \otimes K_{i}^{0}\right)\left(V_{\pi}\right)_{23}\left(\xi_{0} \otimes 1\right) .
$$

It follows from Remark 3.6 that the closure of $\mathcal{T}_{i}$ in $\mathrm{L}^{2}(P)$ is an $N$ - $N$-bimodule isomorphic with $H\left(\psi_{\eta}\right) \otimes_{N} K_{i} \otimes_{N} H\left(\psi_{\pi}\right)$. Note for later use that the ultraweak closure of $P \mathcal{T}_{i}$ is of the form $P q$ for some non-zero projection $q \in P$ that commutes with $N$ and hence equals 1 . So, $P \mathcal{T}_{i}$ is ultraweakly dense in $P$.

We claim that the subspaces of $\mathcal{H}$ defined by $\mathcal{T}_{i} \cdot \mathcal{L} \cdot P, i \in I$, are non-zero and mutually orthogonal in $\mathcal{H}$. Once this claim is proven, we have found inside $\mathcal{H}$ infinitely many orthogonal, non-zero $N-P$ subbimodules. This is a contradiction with ${ }_{P} \mathcal{H}_{P}$ being of finite index and $N \subset P$ being irreducible. So, to conclude step 3, it remains to prove the claim. 
Fix $i \in I$. Since $P \mathcal{T}_{i}$ is ultraweakly dense in $P$, it follows that $\mathcal{T}_{i} \cdot \mathcal{L} \cdot P$ is non-zero. As at the end of Remark 3.6, decompose $\mathrm{L}^{2}(P)$ as a direct sum of irreducible, finite index $N$ - $N$-subbimodules $H_{k}$ such that, writing $H_{k}^{0}:=H_{k} \cap P$, the linear span of all $H_{k}^{0}$ is an ultraweakly dense $*$-subalgebra of $P$. Take $i \neq j$ and take $k, l$. We have to prove that $\mathcal{T}_{i} \cdot \mathcal{L} \cdot H_{k}^{0}$ is orthogonal to $\mathcal{T}_{j} \cdot \mathcal{L} \cdot H_{l}^{0}$. It suffices to prove that their closures are disjoint $N$ - $N$-bimodules.

By Lemma 2.2 and our description above of the closure of $\mathcal{T}_{i}$, the closure of $\mathcal{T}_{i} \cdot \mathcal{L} \cdot H_{k}^{0}$ is isomorphic with an $N$-N-subbimodule of

$$
H\left(\psi_{\eta}\right) \otimes_{N} K_{i} \otimes_{N} H\left(\psi_{\pi}\right) \otimes_{N} \mathcal{L} \otimes_{N} H_{k}
$$

Since $\mathcal{L} \in \mathcal{F} \backslash \mathcal{F}_{0}$, Frobenius reciprocity combined with Remark 3.6, implies that $\mathcal{L} \otimes_{N} H_{k}$ is isomorphic with a direct sum of $N$-N-bimodules of the form

$$
\mathcal{L}^{\prime} \otimes_{N} H\left(\psi_{\pi_{1}}\right) \otimes_{N} R_{1} \otimes_{N} \cdots \otimes_{N} R_{n-1} \otimes_{N} H\left(\psi_{\pi_{n}}\right) \otimes_{N} R_{n}
$$

where $\mathcal{L}^{\prime} \in \mathcal{F} \backslash \mathcal{F}_{0}, n \in \mathbb{N} \cup\{0\}, \pi_{1}, \ldots, \pi_{n} \in \operatorname{Irr}(G) \backslash\{\epsilon\}, R_{1}, \ldots, R_{n}$ are irreducible $N$ - $N$ subbimodules of $\mathrm{L}^{2}\left(M_{0}\right)$ and $R_{1}, \ldots, R_{n-1}$ are non-trivial. Hence, the closure of $\mathcal{T}_{i} \cdot \mathcal{L} \cdot H_{k}^{0}$ is isomorphic with a direct sum of irreducible $N$-N-subbimodules that are of the form

$$
H\left(\psi_{\eta}\right) \otimes_{N} K_{i} \otimes_{N} H\left(\psi_{\pi}\right) \otimes_{N} \mathcal{L}^{\prime} \otimes_{N} H\left(\psi_{\pi_{1}}\right) \otimes_{N} \cdots
$$

with $\mathcal{L}^{\prime} \in \mathcal{F} \backslash \mathcal{F}_{0}$. The freeness of $\mathcal{F}$ and $\operatorname{Rep}(G)$ inside $\operatorname{FAlg}(N)$ implies that two $N$ - $N$-bimodules of this last form, for different values of $i$, can never be isomorphic. This proves the claim.

End of the proof. By Step $3, \mathcal{K}$ contains a non-zero $N$-N-subbimodule that belongs to $\mathcal{F}_{0}$. Hence, assumption 2.b says that $M_{0} \mathcal{K}_{M_{0}}$ is the trivial $M_{0}-M_{0}$-bimodule.

Lemma 3.10. The von Neumann algebra $P$ is generated by $\left\{\left(\xi^{*} \otimes 1\right) \psi_{\pi}\left(M_{0}\right)(\eta \otimes 1) \mid \pi \in\right.$ $\left.\operatorname{Irr}(G), \xi, \eta \in H_{\pi}\right\}$.

Proof. Denote by $P_{0}$ the von Neumann subalgebra of $P$ generated by $\left\{\left(\xi^{*} \otimes 1\right) \psi_{\pi}\left(M_{0}\right)(\eta \otimes 1) \mid \pi \in\right.$ $\left.\operatorname{Irr}(G), \xi, \eta \in H_{\pi}\right\}$. Taking $\pi=\epsilon$, note that $M_{0} \subset P_{0}$. We have to prove that $P_{0}=P$.

By construction (cf. Remark 3.6), the von Neumann algebra $P$ is densely spanned by

$$
\left\{\left(\xi^{*} \otimes 1\right)\left(a_{0}\right)_{n+1}\left(V_{\pi_{1}}\right)_{1, n+1} \cdots\left(V_{\pi_{n}}\right)_{n, n+1}\left(a_{n}\right)_{n+1}(\eta \otimes 1) \mid \xi \in \bigotimes_{i=1}^{n} H_{\pi_{i}}, \eta \in\left(\bigotimes_{i=1}^{n} H_{\pi_{i}}\right)^{G}\right\}
$$

where $a_{0}, \ldots, a_{n} \in M_{0}, \pi_{1}, \ldots, \pi_{n} \in \operatorname{Irr}(G)$ and where the superscript ${ }^{G}$ denotes the subspace of $G$-invariant vectors under the tensor product representation.

It therefore suffices to prove by induction on $n$ that for all $\pi_{1}, \ldots, \pi_{n} \in \operatorname{Irr}(G), \eta \in\left(\bigotimes_{i=1}^{n} H_{\pi_{i}}\right)^{G}$ and $a_{0}, \ldots, a_{n} \in M_{0}$, we have

$$
A_{n}:=\left(a_{0}\right)_{n+1}\left(V_{\pi_{1}}\right)_{1, n+1} \cdots\left(V_{\pi_{n}}\right)_{n, n+1}\left(a_{n}\right)_{n+1}(\eta \otimes 1) \in H_{\pi_{1}} \otimes \cdots \otimes H_{\pi_{n}} \otimes P_{0} .
$$

The case $n=1$ being trivial, assume that (7) holds for all $n \leq k-1$. Take $A_{k}$ as in (7) and re-write $A_{k}$ in the following way.

$$
A_{k}=\left(a_{0}\right)_{k+1}\left(\psi_{\pi_{1}}\left(a_{1}\right)\right)_{1, k+1}\left(V_{\pi_{1}}\right)_{1, k+1}\left(V_{\pi_{2}}\right)_{2, k+1} \cdots\left(a_{k}\right)_{k+1}(\eta \otimes 1) .
$$


Lemma 2.6 yields $\mu_{1}, \ldots, \mu_{r} \in \operatorname{Irr}(G)$, isometries $v_{i} \in \operatorname{Mor}\left(\mu_{i}, \pi_{1} \otimes \pi_{2}\right)$ and $X_{i} \in \mathrm{B}\left(H_{\mu_{i}}, H_{\pi_{1}} \otimes\right.$ $\left.H_{\pi_{2}}\right) \otimes N$ such that

$$
\left(V_{\pi_{1}}\right)_{13}\left(V_{\pi_{2}}\right)_{23}=\sum_{i=1}^{r} X_{i} V_{\mu_{i}}\left(v_{i}^{*} \otimes 1\right) .
$$

Put $\xi_{i}:=\left(v_{i}^{*}\right)_{12} \eta \in\left(H_{\mu_{i}} \otimes H_{\pi_{3}} \otimes \cdots \otimes H_{\pi_{n}}\right)^{G}$ and

$$
B_{i}:=\left(V_{\mu_{i}}\right)_{1, n}\left(a_{2}\right)_{n}\left(V_{\pi_{3}}\right)_{2, n}\left(V_{\pi_{n}}\right)_{n-1, n}\left(a_{n}\right)_{n}\left(\xi_{i} \otimes 1\right) .
$$

By the induction hypothesis, $B_{i} \in H_{\mu_{i}} \otimes \bigotimes_{i=3}^{n} H_{\pi_{i}} \otimes P_{0}$, for all $i$. Also, $a_{0} \in P_{0}$ and $\psi_{\pi_{1}}\left(a_{1}\right) \in$ $\mathrm{B}\left(H_{\pi_{1}}\right) \otimes P_{0}$. Since $X_{i} \in \mathrm{B}\left(H_{\mu_{i}}, H_{\pi_{1}} \otimes H_{\pi_{2}}\right) \otimes P_{0}$, it follows that

$$
A_{k}=\left(a_{0}\right)_{k+1}\left(\psi_{\pi_{1}}\left(a_{1}\right)\right)_{1, k+1} \sum_{i=1}^{r}\left(X_{i}\right)_{1,2, k+1} B_{i} \in H_{\pi_{1}} \otimes \cdots \otimes H_{\pi_{k}} \otimes P_{0} .
$$

So, the lemma is proven.

We can finally prove Theorem 3.5.

Proof. Since the functor

$$
\operatorname{Rep}(G) \rightarrow \operatorname{Bimod}(P): \pi \mapsto{ }_{P} \operatorname{Mor}\left(H_{\pi}, \mathrm{L}^{2}(M)\right)_{P}
$$

is a fully faithful tensor functor, it suffices to prove that for every irreducible finite index $P$ - $P$ bimodule ${ }_{P} \mathcal{H}_{P}$, there exists an $\eta \in \operatorname{Irr}(G)$ such that ${ }_{P} \mathcal{H}_{P} \cong{ }_{\psi_{\eta}(P)}\left(H_{\eta} \otimes \mathrm{L}^{2}(P)\right)_{P}$. So, let ${ }_{P} \mathcal{H}_{P}$ be an irreducible finite index $P$ - $P$-bimodule.

Decompose $\mathcal{H}$ into a direct sum $\mathcal{H}=\bigoplus_{i=1}^{k} \mathcal{H}^{i}$ of irreducible $M_{0}$-P-subbimodules. By Lemma 3.8, we can take $\eta_{1}, \ldots, \eta_{k} \in \operatorname{Irr}(G)$ and non-zero irreducible $M_{0}-\psi_{\eta_{i}}\left(M_{0}\right)$-subbimodules $\mathcal{K}^{i} \subset H_{\eta_{i}}^{*} \otimes \mathcal{H}^{i}$ such that $M_{0} \mathcal{K}^{i}{ }_{\eta_{i}\left(M_{0}\right)}$ has finite index. Viewing $\mathcal{K}^{i}$ as an $M_{0}-\psi_{\eta_{i}}\left(M_{0}\right)$-subbimodule of the finite index bimodule ${ }_{P}\left(H_{\eta_{i}}^{*} \otimes \mathcal{H}\right)_{\psi_{\eta_{i}}(P)}$, Lemma 3.9 says that ${ }_{M_{0}} \mathcal{K}^{i}{ }_{\psi_{\eta_{i}}\left(M_{0}\right)} \cong{ }_{M_{0}} \mathrm{~L}^{2}\left(M_{0}\right)_{M_{0}}$.

Take a finite index inclusion $\psi: P \rightarrow p P^{n} p$ such that ${ }_{P} \mathcal{H}_{P} \cong{ }_{\psi(P)} p\left(\mathbb{C}^{n} \otimes \mathrm{L}^{2}(P)\right)_{P}$. Denote $A=$ $\psi\left(M_{0}\right)^{\prime} \cap p P^{n} p$. Then, $A$ is finite dimensional and $\mathcal{H}^{i}$ corresponds to $p_{i}\left(\mathbb{C}^{n} \otimes \mathrm{L}^{2}(P)\right)$, where $p_{1}, \ldots, p_{k}$ are minimal projections in $A$ summing to 1 . In the previous paragraph, it was shown that $\psi\left(M_{0}\right) p_{i} p_{i}\left(\mathbb{C}^{n} H_{\eta_{i}}^{*} \otimes \mathrm{L}^{2}(P)\right)_{\psi_{\eta_{i}}\left(M_{0}\right)}$ contains the trivial $M_{0}-\psi_{\eta_{i}}\left(M_{0}\right)$-bimodule. So, we can take non-zero vectors $v_{i} \in p_{i}\left(\mathbb{C}^{n} H_{\eta_{i}}^{*} \otimes \mathrm{L}^{2}(P)\right)$ satisfying $\psi(a) v_{i}=v_{i} \psi_{\eta_{i}}(a)$ for all $a \in M_{0}$. As an element of $p_{i}\left(\mathrm{M}_{n}(\mathbb{C}) \otimes \mathrm{L}^{1}(P)\right) p_{i}$, the operator $v_{i} v_{i}^{*}$ commutes with $\psi\left(M_{0}\right)$. By minimality of $p_{i}$, it follows that $v_{i} v_{i}^{*}$ is a multiple of $p_{i}$ and in particular, $v_{i} \in p_{i}\left(\mathbb{C}^{n} H_{\eta_{i}}^{*} \otimes P\right)$. So, we may assume that $v_{i} v_{i}^{*}=p_{i}$. On the other hand, $v_{i}^{*} v_{i} \in \mathrm{B}\left(H_{\eta_{i}}\right) \otimes P \cap \psi_{\eta_{i}}\left(M_{0}\right)^{\prime}=\mathbb{C} 1$, implying that $v_{i}^{*} v_{i}=1$.

Define $\eta=\bigoplus_{i=1}^{k} \eta_{i}$ and put $\psi_{\eta}: P \rightarrow \mathrm{B}\left(H_{\eta}\right) \otimes P: \psi_{\eta}(a)=\bigoplus_{i=1}^{k} \psi_{\eta_{i}}(a)$. Note that $\psi_{\eta}(a)=$ $V_{\eta}(1 \otimes a) V_{\eta}^{*}$, where $V_{\eta}=\bigoplus_{i=1}^{k} V_{\eta_{i}}$. We have shown the existence of $u \in p\left(\mathbb{C}^{n} H_{\eta}^{*} \otimes P\right)$ satisfying $u u^{*}=p, u^{*} u=1$ and $u^{*} \psi(a) u=\psi_{\eta}(a)$ for all $a \in M_{0}$. We may assume from now on that ${ }_{P} \mathcal{H}_{P} \cong$ $\psi(P)\left(H_{\eta} \otimes \mathrm{L}^{2}(P)\right)_{P}$ where $\psi: P \rightarrow \mathrm{B}\left(H_{\eta}\right) \otimes P$ is a finite index inclusion satisfying $\psi(a)=\psi_{\eta}(a)$ for all $a \in M_{0}$. It remains to prove that $\psi(a)=\psi_{\eta}(a)$ for all $a \in P$.

By Lemma 3.10, it suffices to prove that $(\mathrm{id} \otimes \psi) \psi_{\pi}(a)=\left(\mathrm{id} \otimes \psi_{\eta}\right) \psi_{\pi}(a)$ for all $a \in M_{0}$ and all $\pi \in \operatorname{Irr}(G)$. Fix $\pi \in \operatorname{Irr}(G)$. Applying the reasoning in the previous paragraphs to the $P$ - $P$ bimodule $\psi_{\pi}(P)\left(H_{\pi} \otimes \mathcal{H}\right)_{P}$, we find a finite dimensional unitary representation $\gamma$ of $G$ and a unitary $X \in \mathrm{B}\left(H_{\pi} \otimes H_{\eta}, H_{\gamma}\right) \otimes P$ satisfying

$$
(\operatorname{id} \otimes \psi) \psi_{\pi}(a)=X \psi_{\gamma}(a) X^{*}
$$


for all $a \in M_{0}$. Because $\psi_{\pi}(N) \subset \mathrm{B}\left(H_{\pi}\right) \otimes N$ and $\psi(a)=\psi_{\eta}(a)$ for all $a \in N$, it follows in particular that $\left(\mathrm{id} \otimes \psi_{\eta}\right) \psi_{\pi}(a)=X \psi_{\gamma}(a) X^{*}$ for all $a \in N$. Hence, the unitary

$$
Z:=\left(V_{\eta}\right)_{23}^{*}\left(V_{\pi}\right)_{13}^{*} X V_{\gamma}
$$

satisfies $Z(1 \otimes a)=(1 \otimes 1 \otimes a) Z$ for all $a \in N$. So, $Z=U \otimes 1$, where $U: H_{\gamma} \rightarrow H_{\pi} \otimes H_{\eta}$ intertwines the representations $\gamma$ and $\pi \otimes \eta$. It follows that $X \psi_{\gamma}(a) X^{*}=\left(\right.$ id $\left.\otimes \psi_{\eta}\right) \psi_{\pi}(a)$ for all $a \in M_{0}$. Combining with (8), we conclude that $(\operatorname{id} \otimes \psi) \psi_{\pi}(a)=\left(\operatorname{id} \otimes \psi_{\eta}\right) \psi_{\pi}(a)$ for all $a \in M_{0}$.

As a consequence of Theorem 3.5, we now prove Theorem 1.1 stated in the introduction.

Proof of Theorem 1.1. Denote by $M_{1}$ the hyperfinite $\mathrm{II}_{1}$ factor and take a minimal action $G \curvearrowright M_{1}$. Put $N:=M_{1}^{G}$.

Define the group $\Gamma$, its subgroup $\Lambda<\Gamma$ and the scalar 2-cocycle $\Omega \in \mathrm{Z}^{2}\left(\Gamma, S^{1}\right)$ as in Example 3.4. Write $R:=\mathrm{L}_{\Omega}(\Lambda)$ and $M_{0}:=\mathrm{L}_{\Omega}(\Gamma)$. Denote by $\mathcal{F}$ the fusion subalgebra of FAlg $(R)$ generated by all finite index $R$ - $R$-bimodules appearing as an $R$ - $R$-subbimodule of a finite index $M_{0}$ - $M_{0}$-bimodule. By Remark 3.1, $\mathcal{F}$ is countable.

Note that both $N$ and $R$ are isomorphic with the hyperfinite $\mathrm{II}_{1}$ factor. Whenever $\alpha: N \rightarrow R$ is an isomorphism, we can view $\alpha^{-1} \mathcal{F} \alpha$ as a fusion subalgebra of $\operatorname{FAlg}(N)$. By Theorem 2.8, we can choose $\alpha$ such that $\alpha^{-1} \mathcal{F} \alpha$ is free w.r.t. the image of $\operatorname{Rep}(G)$ inside $\operatorname{FAlg}(N)$. Identifying $N$ and $R$ through $\alpha$, it follows from Example 3.4 that all assumptions for Theorem 3.5 are satisfied.

So, we can take $M:=M_{0} *_{N} M_{1}$, extend $G \curvearrowright M_{1}$ to a minimal action $G \curvearrowright M$ by acting trivially on $M_{0}$ and conclude from Theorem 3.5 that the natural tensor functor $\operatorname{Rep}(G) \rightarrow \operatorname{Bimod}\left(M^{G}\right)$ is an equivalence of categories.

\section{Proof of Theorem 1.2}

Fix a second countable compact group $G$ and a minimal action $G \stackrel{\sigma}{\curvearrowright} M$ on a $\mathrm{II}_{1}$ factor $M$. Let $G \stackrel{\alpha}{\curvearrowright} A$ be an action of $G$ on the finite dimensional von Neumann algebra $A$. Denote by $\alpha \otimes \sigma$ the diagonal action of $G$ on $A \otimes M$, given by $(\alpha \otimes \sigma)_{g}=\alpha_{g} \otimes \sigma_{g}$ for all $g \in G$. Denote by $N^{\beta}$ the fixed point algebra of an action $\beta$ on a von Neumann algebra $N$.

Lemma 4.1. The fixed point algebra $(A \otimes M)^{\alpha \otimes \sigma}$ is a factor iff $\mathcal{Z}(A)^{\alpha}=\mathbb{C} 1$. The inclusion $1 \otimes M^{\sigma} \subset(A \otimes M)^{\alpha \otimes \sigma}$ is irreducible iff $A^{\alpha}=\mathbb{C} 1$.

Every intermediate von Neumann algebra $1 \otimes M^{\sigma} \subset N \subset(A \otimes M)^{\alpha \otimes \sigma}$ is of the form $(D \otimes M)^{\alpha \otimes \sigma}$ for a uniquely determined globally $G$-invariant $*$-subalgebra $D \subset A$.

Proof. Denote $P:=M^{\sigma}$. By minimality, the relative commutant of $1 \otimes P$ inside $(A \otimes M)^{\alpha \otimes \sigma}$ equals $A^{\alpha} \otimes 1$. So, the inclusion $1 \otimes P \subset(A \otimes M)^{\alpha \otimes \sigma}$ is irreducible iff $A^{\alpha}=\mathbb{C} 1$. Also, $\mathcal{Z}\left((A \otimes M)^{\alpha \otimes \sigma}\right) \subset$ $A^{\alpha} \otimes 1$.

We claim that

$$
A=\operatorname{span}\left\{(\operatorname{id} \otimes \omega)(a) \mid a \in(A \otimes M)^{\alpha \otimes \sigma}, \omega \in M_{*}\right\} .
$$

In order to prove this claim, let $\pi \in \operatorname{Irr}(G), X \in H_{\pi}^{*} \otimes A$ and (id $\left.\otimes \alpha_{g}\right)(X)=X(\pi(g) \otimes 1)$ for all $g \in G$. Note that $A$ is the linear span of all possible $X\left(H_{\pi} \otimes 1\right)$. On the other hand, $X_{12}\left(V_{\pi}\right)_{13}^{*}$ belongs to $H_{\pi}^{*} \otimes(A \otimes M)^{\alpha \otimes \sigma}$, implying that $X\left(H_{\pi} \otimes 1\right)$ is included in the expression at the right-hand side of (9). This proves (9). 
A combination of the claim and the first paragraph of the proof implies that $\mathcal{Z}\left((A \otimes M)^{\alpha \otimes \sigma}\right)=$ $\mathcal{Z}(A)^{\alpha} \otimes 1$. Hence, $(A \otimes M)^{\alpha \otimes \sigma}$ is a factor iff $\mathcal{Z}(A)^{\alpha}=\mathbb{C} 1$.

Let $1 \otimes M^{\sigma} \subset N \subset(A \otimes M)^{\alpha \otimes \sigma}$ be an intermediate von Neumann algebra. Choose a $G$-invariant trace on $A$, so that we can view $A$ as a finite-dimensional Hilbert space. In this way, the action $\alpha$ can be seen as a unitary representation $\pi_{A}: G \rightarrow \mathcal{U}(A)$. Choose a unitary $W \in \mathrm{B}(A) \otimes M$ satisfying (id $\left.\otimes \sigma_{g}\right)(W)=W\left(\pi_{A}(g) \otimes 1\right)$ for all $g \in G$. Define the finite index inclusion $\gamma: P \rightarrow$ $\mathrm{B}(A) \otimes P: \gamma(a)=W(1 \otimes a) W^{*}$. The map $a \mapsto W a$ defines a $P$-P-bimodular unitary

$$
\Theta:(1 \otimes P)^{2} \mathrm{~L}^{2}\left((A \otimes M)^{\alpha \otimes \sigma}\right)_{(1 \otimes P)} \rightarrow \gamma(P)\left(A \otimes \mathrm{L}^{2}(P)\right)_{P} .
$$

It follows that $\Theta(N)=q\left(A \otimes \mathrm{L}^{2}(P)\right)$, where $q$ is a projection in

$$
\mathrm{B}(A) \otimes P \cap \gamma(P)^{\prime}=W\left(\mathrm{~B}(A)^{\mathrm{Ad} \pi_{A}} \otimes 1\right) W^{*} .
$$

Write $q=W(p \otimes 1) W^{*}$ and define the vector subspace $D \subset A$ as the image of the projection $p$. Since $p$ commutes with $\pi_{A}(G)$, it follows that $D$ is globally $\alpha$-invariant. We have shown that

$$
N=(A \otimes M)^{\alpha \otimes \sigma} \cap(D \otimes M)=(D \otimes M)^{\alpha \otimes \sigma} .
$$

It remains to prove that $D$ is a $*$-algebra.

Since $D$ is globally $\alpha$-invariant, it follows that $D$ is linearly spanned by elements of the form $X\left(H_{\pi} \otimes 1\right)$, where $\pi \in \operatorname{Irr}(G), X \in H_{\pi}^{*} \otimes D$ and (id $\left.\otimes \alpha_{g}\right)(X)=X(\pi(g) \otimes 1)$ for all $g \in G$. As in (9), it follows that $D$ is linearly spanned by $(\mathrm{id} \otimes \omega)(a), \omega \in M_{*}$ and $a \in N$. Hence, $D=D^{*}$.

Further, let $\pi, \eta \in \operatorname{Irr}(G), X \in H_{\pi}^{*} \otimes D, Y \in H_{\eta}^{*} \otimes D$ and (id $\left.\otimes \alpha_{g}\right)(X)=X(\pi(g) \otimes 1)$, (id $\otimes$ $\left.\alpha_{g}\right)(Y)=Y(\eta(g) \otimes 1)$ for all $g \in G$. To conclude the proof of the lemma, it suffices to show that $X_{13} Y_{23} \in\left(H_{\pi} \otimes H_{\eta}\right)^{*} \otimes D$. But, we know that $X_{12}\left(V_{\pi}\right)_{13}^{*} \in H_{\pi}^{*} \otimes N$ and $Y_{12}\left(V_{\eta}\right)_{13}^{*} \in H_{\eta}^{*} \otimes N$. Since $N$ is an algebra, it follows that

$$
X_{13}\left(V_{\pi}\right)_{14}^{*} Y_{23}\left(V_{\eta}\right)_{24}^{*} \in\left(H_{\pi} \otimes H_{\eta}\right)^{*} \otimes N \subset\left(H_{\pi} \otimes H_{\eta}\right)^{*} \otimes D \otimes M .
$$

The two factors in the middle commute and the conclusion follows.

We are now ready to prove Theorem 1.2.

Proof of Theorem 1.2. Take $G \curvearrowright M$ as in the formulation of the theorem and put $P:=M^{\sigma}$. Let $P_{0} \subset P$ be a finite index subfactor. We first prove that $P_{0}$ is unitarily conjugate in $P$ to a subfactor of the form $P(\alpha)$ for some action $G \stackrel{\alpha}{\curvearrowright} A$ of $G$ on a finite dimensional von Neumann algebra $A$ satisfying $\mathcal{Z}(A)^{\alpha}=\mathbb{C} 1$.

Let $P_{0} \subset P \subset P_{1}$ be the basic construction. Then, ${ }_{P} \mathrm{~L}^{2}\left(P_{1}\right)_{P}$ is a finite index $P$ - $P$-bimodule. By assumption, we find a finite dimensional unitary representation $\pi: G \rightarrow \mathcal{U}(n)$ and a unitary $V \in \mathrm{M}_{n}(\mathbb{C}) \otimes M$ satisfying $\left(\mathrm{id} \otimes \sigma_{g}\right)(V)=V(\pi(g) \otimes 1)$ for all $g \in G$, such that

$$
{ }_{P} \mathrm{~L}^{2}\left(P_{1}\right)_{P} \cong{ }_{\gamma(P)}\left(\mathbb{C}^{n} \otimes \mathrm{L}^{2}(P)\right)_{P} \quad \text { where } \quad \gamma(a)=V(1 \otimes a) V^{*} \text { for all } a \in P .
$$

The left $P_{1}$-action on $\mathrm{L}^{2}\left(P_{1}\right)$ commutes with the right $P$-action and hence, we can extend $\gamma$ to an inclusion $\gamma: P_{1} \rightarrow \mathrm{M}_{n}(\mathbb{C}) \otimes P$. Denote $N=V^{*} \gamma\left(P_{1}\right) V$ and write $\alpha(g)=\operatorname{Ad}(\pi(g))$. It follows that $1 \otimes P \subset N \subset\left(\mathrm{M}_{n}(\mathbb{C}) \otimes M\right)^{\alpha \otimes \sigma}$. Applying Lemma 4.1, we find a finite dimensional von Neumann algebra $A$, an action $G \stackrel{\alpha}{\curvearrowright} A$ satisfying $\mathcal{Z}(A)^{\alpha}=\mathbb{C} 1$ and a *-isomorphism $\theta: P_{1} \rightarrow(A \otimes M)^{\alpha \otimes \sigma}$ 
satisfying $\theta(a)=1 \otimes a$ for all $a \in P$. By uniqueness of the tunnel construction, it follows that $P_{0}$ and $P(\alpha)$ are unitarily conjugate.

Finally, suppose that $G \stackrel{\alpha}{\curvearrowright} A$ and $G \stackrel{\beta}{\curvearrowright} B$ satisfy $\mathcal{Z}(A)^{\alpha}=\mathbb{C} 1$ and $\mathcal{Z}(B)^{\beta}=1$ and suppose that the subfactors $P(\alpha)$ and $P(\beta)$ are unitarily conjugate in $P$. It remains to construct a $*$-isomorphism $\pi: A \rightarrow B$ satisfying $\beta_{g} \circ \pi=\pi \circ \alpha_{g}$ for all $g \in G$. By assumption, we find a $*$-isomorphism $\theta:(A \otimes M)^{\alpha \otimes \sigma} \rightarrow(B \otimes M)^{\beta \otimes \sigma}$ satisfying $\theta(1 \otimes a)=1 \otimes a$ for all $a \in P$. Repeating the argument given in the proof of Lemma 4.1, we find a bijective linear map $\gamma: A \rightarrow B$ such that $\gamma \circ \alpha_{g}=\beta_{g} \circ \gamma$ for all $g \in G$ and $\theta(b)=(\gamma \otimes \mathrm{id})(b)$ for all $b \in(A \otimes M)^{\alpha \otimes \sigma}$. By (9), it follows that $\gamma$ is a *-isomorphism.

\section{References}

[1] D. Bisch, Bimodules, higher relative commutants and the fusion algebra associated to a subfactor. In Operator algebras and their applications, Fields Inst. Commun. 13, Amer. Math. Soc., Providence, RI, 1997, pp. 13-63.

[2] D. Bisch and V.F.R. Jones, Algebras associated to intermediate subfactors. Invent. Math. 128 (1997), 89-157.

[3] A. Connes, Noncommutative Geometry. Academic Press, 1994.

[4] A. Connes And V.F.R Jones, Property (T) for von Neumann algebras. Bull. London Math. Soc. 17 (1985), 57-62.

[5] S. FalguiÈres And S. VAes, Every compact group arises as the outer automorphism group of a $\mathrm{II}_{1}$ factor. J. Func. Anal. 254 (2008), 2317-2328.

[6] C. Houdayer, Construction of type $\mathrm{II}_{1}$ factors with prescribed countable fundamental group. To appear in J. Reine Angew. Math. arXiv:0704.3502

[7] A. Ionna, J. Peterson and S. Popa, Amalgamated free products of $w$-rigid factors and calculation of their symmetry group. Acta Math. 200 (2008), no. 1, 85-153.

[8] V.F.R Jones, Index of subfactors. Invent. Math. 72 (1983), 1-25.

[9] F.J. Murray and J. von Neumann, On rings of operators. Ann. of Math. (2) 37 (1936), 116-229.

[10] S. PopA, Strong rigidity of $\mathrm{II}_{1}$ factors arising from malleable actions of $w$-rigid groups, Part I. Invent. Math. 165 (2006), 369-408.

[11] S. PopA, Strong rigidity of $\mathrm{II}_{1}$ factors arising from malleable actions of $w$-rigid groups, Part II. Invent. Math. 165 (2006), 409-451.

[12] S. PopA, On a class of type $\mathrm{II}_{1}$ factors with Betti numbers invariants. Ann. Math. 163 (2006), 809-899.

[13] S. Popa, Free-independent sequences in type $\mathrm{II}_{1}$ factors and related problems. Astérisque 232 (1995), 187-202.

[14] S. Popa, Markov traces on universal Jones algebras and subfactors of finite index. Invent. Math. 111 (1993), 375-405.

[15] S. Popa, Correspondences. INCREST Preprint (1986).

[16] S. Popa AND S. VAES, On the fundamental group of $\mathrm{II}_{1}$ factors and equivalence relations arising from group actions. Preprint. arXiv:0810.0706

[17] S. Popa AND S. VAes, Actions of $\mathbb{F}_{\infty}$ whose $\mathrm{II}_{1}$ factors and orbit equivalence relations have prescribed fundamental group. Preprint. arXiv:0803.3351

[18] S. Popa And S. Vaes, Strong rigidity of generalized Bernoulli actions and computations of their symmetry groups. Adv. Math. 217 (2008), 833-872.

[19] J.E. Roberts, Cross products of von Neumann algebras by group duals. In Symposia Mathematica, Vol. XX, Academic Press, London, 1976, pp. 335-363.

[20] S. VAEs, Explicit computations of all finite index bimodules for a family of $\mathrm{II}_{1}$ factors. Ann. Sci. École Norm. Sup. 41 (2008), 743-788. 
[21] S. Vaes, Factors of type $\mathrm{II}_{1}$ without non-trivial finite index. Trans. Amer. Math. Soc. 361 (2009), 2587-2606.

[22] S. VAes, Rigidity results for Bernoulli actions and their von Neumann algebras (after Sorin Popa). Séminaire Bourbaki, exp. no. 961. Astérisque 311 (2007), 237-294.

[23] D.V. Voiculescu, K.J. Dykema and A. Nica, Free random variables. CRM Monograph Series 1, American Mathematical Society, Providence, RI, 1992.

[24] A. Wassermann, Ergodic Actions of Compact Groups on Operator Algebras: I. General Theory. Ann. Math. 130 (1989), 273-319. 